\title{
Upper Semicontinuity of Random Attractors for Nonautonomous Stochastic Reversible Selkov System with Multiplicative Noise
}

\author{
Chunxiao Guo $\mathbb{D}^{1},{ }^{1}$ Yanfeng Guo $\mathbb{D},^{2,3}$ and Xiaohan $\mathrm{Li}^{1}$ \\ ${ }^{1}$ Department of Mathematics, China University of Mining and Technology, Beijing, Beijing 100083, China \\ ${ }^{2}$ School of Science, Guangxi University of Science and Technology, Liuzhou, Guangxi 545006, China \\ ${ }^{3}$ School of Mathematics and Physics, China University of Geosciences, Wuhan, Hubei 430074, China \\ Correspondence should be addressed to Yanfeng Guo; guoyan_feng@163.com
}

Received 23 April 2019; Accepted 12 June 2019; Published 27 June 2019

Academic Editor: Ciprian G. Gal

Copyright (C) 2019 Chunxiao Guo et al. This is an open access article distributed under the Creative Commons Attribution License, which permits unrestricted use, distribution, and reproduction in any medium, provided the original work is properly cited.

In this paper, the existence of random attractors for nonautonomous stochastic reversible Selkov system with multiplicative noise has been proved through Ornstein-Uhlenbeck transformation. Furthermore, the upper semicontinuity of random attractors is discussed when the intensity of noise approaches zero. The main difficulty is to prove the asymptotic compactness for establishing the existence of tempered pullback random attractor.

\section{Introduction}

Selkov equations [1] were a system of ODEs originally proposed by Selkov $[2,3]$ as a simplified model of a biochemical process called glycolysis, through which living cells get energy from breaking down sugar. The existence and robustness of global attractors of the reversible Selkov system have been investigated in the deterministic case (i.e., $\sigma=0$ ) by You in [4]. Li proved the existence of random attractor of the stochastic reversible Selkov system on infinite lattice with additive noise in [5]. In this paper, we consider the upper semicontinuity of random attractors for nonautonomous stochastic reversible Selkov system with multiplicative noise

$$
\begin{aligned}
d u= & \left(d_{1} \Delta u+\rho-a u+u^{2} v-G u^{3}+f_{1}(t, x)\right) d t+\sigma u \\
& \circ d W(t), \\
d v= & \left(d_{2} \Delta v+\beta-b v-u^{2} v+G u^{3}+f_{2}(t, x)\right) d t+\sigma v \\
& \circ d W(t),
\end{aligned}
$$

where $\Gamma \subset \mathbf{R}^{n}(n \leq 3)$ is a bounded smooth domain. There are boundary conditions

$$
u(t, x)=v(t, x)=0, \quad x \in \partial \Gamma,
$$

and initial conditions

$$
\begin{aligned}
& u(\tau, x)=u_{\tau}(x), \\
& v(\tau, x)=v_{\tau}(x) .
\end{aligned}
$$

All the coefficients $d_{1}, d_{2}, \rho, \beta, a, b$, and $G$ are arbitrarily given positive constants; $f_{i}(i=1,2)$ denote the time-dependent external forces. $\{W(t)\}_{t \in \mathbf{R}}$ is a one-dimensional two-sided standard Wiener process on a probability space. The terms $\sigma u \circ d W(t), \sigma v \circ d W(t)$ indicate that (1)-(2) are interpreted in the sense of the Stratonovich integration.

In the past decades, great progress has been made in various aspects of random dynamical systems; see [6-22]. The long-time behavior of solutions for nonautonomous threecomponent reversible Gray-Scott system was discussed in [23] and the existence and upper semicontinuity of random attractor for stochastic three-component reversible GrayScott system was proved in [24]. In [25], Tu and You introduced the pullback asymptotic compactness of stochastic Brusselator system with multiplicative noise. Notice that (1) 
and (2) are nonautonomous stochastic equations with timedependent external terms $f_{1}$ and $f_{2}$. For such a nonautonomous stochastic system, Wang established an efficacious theory about the existence and upper semicontinuity of the random attractor by introducing two parametric spaces [2629].

In this paper, we first obtain the existence of random attractors for the stochastic perturbed reversible Selkov system (1)-(4) defined on $\Gamma \subset \mathbf{R}^{n}(n \leq 3)$. When $\sigma \longrightarrow 0$, we will consider the limit behaviors of random attractors and prove the upper semicontinuity of these perturbed random attractors. As usual, we must prove the pullback asymptotic compactness of the solution operators in $L^{2}(\Gamma)$. The main difficulty for proving such compactness lines is the uniform estimates of the solutions in $\left[L^{6}(\Gamma)\right]^{2}$ when we need to obtain some estimates in $\left[H^{1}(\Gamma)\right]^{2}$. We obtain the important $L^{6}(\Gamma)$ norm estimates by using the Mean Value Theorem.

The outline of the paper is as follows. In Section 2, some basic concepts related to the nonautonomous random dynamical system and upper semicontinuity of random attractors are introduced. Section 3 is devoted to the pullback asymptotic compactness and the existence of random attractors. In Section 4, we establish the upper semicontinuity of random attractors when the coefficient $\sigma$ approaches zero.

\section{Preliminaries}

In this section, we use some concepts of nonautonomous random dynamical system and random attractor. Let $(X, \|$. $\|_{X}$ ) be a real separable Banach space with Borel $\sigma$-algebra $\mathscr{B}(X)$ and $\left(\Omega, \mathscr{F}, P,\left(\theta_{t}\right)_{t \in \mathbf{R}}\right)$ be an ergodic metric dynamical system. Some concepts and definitions of continuous random dynamical system have been seen in $[6,8,17,30]$ for more details. The following theorem is used to obtain the existence of random attractors for the continuous random dynamical system.

Theorem 1 (see $[6,8])$. Let $\varphi$ be a continuous random dynamical system on $X$ over $\left(\Omega, \mathscr{F}, P,\left(\theta_{t}\right)_{t \in \mathbf{R}}\right)$. If there exists a closed random tempered absorbing $\operatorname{set}\{B(\tau, \omega): \tau \in \mathbf{R}, \omega \in \Omega\}$ of $\varphi$ and $\varphi$ is asymptotically compact in $X$, then $\mathscr{A}(\tau, \omega)$ is a random attractor of $\varphi$, where

$$
\begin{array}{r}
\mathscr{A}(\tau, \omega)=\bigcap_{r \geq 0} \varphi \bigcup_{t \geq r}\left(t, \tau-t, \theta_{-t} \omega, B\left(\tau-t, \theta_{-t} \omega\right)\right) \\
\tau \in \mathbf{R}, \omega \in \Omega .
\end{array}
$$

Moreover, $\mathscr{A}(\tau, \omega)$ is the random attractor of $\varphi$.

Now, we define the product Hilbert spaces

$$
\begin{aligned}
& H=\left[L^{2}(\Gamma)\right]^{2}, \\
& E=\left[H_{0}^{1}(\Gamma)\right]^{2} .
\end{aligned}
$$

The norm and inner-product in $H$ or $L^{2}(\Gamma)$ will be denoted by $\|\cdot\|$ and $(\cdot, \cdot)$, respectively. The norm in $L^{p}(\Gamma)$ or $\left[L^{p}(\Gamma)\right]^{2}$ will be denoted by $\|\cdot\|_{L^{p}}$, if $p \neq 2$. The norm in $E$ will be denoted by $\|\cdot\|_{E}$.
Let $\{W(t)\}_{t \in \mathbf{R}}$ be the standard one-dimensional, twosided Wiener process in the complete probability space $(\Omega, \mathscr{F}, P)$, where

$$
\Omega=\{\omega \in C(\mathbf{R}, \mathbf{R}): \omega(0)=0\},
$$

the $\sigma$-algebra $\mathscr{F}$ is generated by the compact-open topology on $\Omega$, and $P$ is the corresponding Wiener measure on $\mathscr{F}$; see $[6,8]$. The Wiener shift $\theta_{t}$ is defined by

$$
\theta_{t} \omega(\cdot)=\omega(\cdot+t)-\omega(t), \quad \omega \in \Omega, t \in \mathbf{R} .
$$

Consider the Ornstein-Uhlenbeck process

$$
\begin{aligned}
z\left(\theta_{t} \omega\right) & :=-\int_{-\infty}^{0} e^{s}\left(\theta_{t} \omega\right)(s) d \omega \\
& =-\int_{-\infty}^{0} e^{s} \omega(t+s) d s+\omega(t)
\end{aligned}
$$

which solves the Itô equation

$$
d z+z d t=d W(t)
$$

Proposition 2 (see $[6,8]$ ). Let the metric dynamical system $\left(\Omega, \mathscr{F}, P,\left(\theta_{t}\right)_{t \in \mathbf{R}}\right)$ and the Ornstein-Uhlenbeck process $\left\{z\left(\theta_{t} \omega\right)\right\}_{t \in \mathbf{R}}$ be defined as above. There is a $\theta_{t}$-invariant set $\widetilde{\Omega} \in \Omega$ of full P-measure such that for every $\omega \in \widetilde{\Omega}$, the following statements hold:

(1) The Ornstein-Uhlenbeck process $\left\{z\left(\theta_{t} \omega\right)\right\}_{t \in \mathbf{R}}$ has the asymptotically sublinear growth property; i.e.,

$$
\lim _{t \rightarrow \pm \infty} \frac{\left|z\left(\theta_{t} \omega\right)\right|}{|t|}=0 .
$$

(2) $z\left(\theta_{t} \omega\right)$ is continuous in $t$ and, for any fixed $t_{0} \in \mathbf{R}$,

$$
\lim _{t \rightarrow \pm \infty} \frac{1}{t-t_{0}} \int_{t_{0}}^{t} z\left(\theta_{s} \omega\right) d s=0 .
$$

In the sequel, we consider $\omega \in \widetilde{\Omega}$ only and will always write $\Omega$ for $\widetilde{\Omega}$.

Proposition 3 ((upper semicontinuity of random attractors) $($ see $[8,28]))$. Suppose the following conditions are satisfied:

(1) Given $\sigma>0$, we suppose that $\varphi_{\sigma}$ is a random dynamical system over a metric system $\left(\Omega, \mathscr{F}, P,\left(\theta_{t}\right)_{t \in \mathbf{R}}\right)$. For $P$-a.e. $\tau \in \mathbf{R}$, $\omega \in \Omega, t \geq 0, \sigma_{n} \longrightarrow 0$, and $x_{n}, x \in X$ with $x_{n} \longrightarrow x$, there holds

$$
\lim _{n \longrightarrow \pm \infty} \varphi_{\sigma}\left(t, \tau, \omega, x_{n}\right)=\varphi(t, \tau) x
$$

(2) Assume that $\varphi_{\sigma}$ has a random attractor $\mathscr{A}_{\sigma}(\tau, \omega)=$ $\left\{\mathscr{A}_{\sigma}(\tau, \omega): \tau \in \mathbf{R}, \omega \in \Omega\right\} \in \mathscr{D}$ and a random absorbing set $K_{\sigma}(\tau, \omega)=\left\{K_{\sigma}(\tau, \omega): \tau \in \mathbf{R}, \omega \in \Omega\right\} \in \mathscr{D}$ such that for some deterministic positive constant $c$ and for P-a.e. $\tau \in \mathbf{R}, \omega \in \Omega$

$$
\limsup _{\sigma \rightarrow 0}\left\|K_{\sigma}(\tau, \omega)\right\|_{X} \leq c
$$

where $\left\|K_{\sigma}(\tau, \omega)\right\|_{X}=\sup _{x \in K_{\sigma}(\tau, \omega)}\|x\|_{X}$. 
(3) There exists a $\sigma_{0}>0$ such that for $P$-a.e. $\tau \in \mathbf{R}, \omega \in \Omega$

$$
\bigcup_{0<\sigma \leq \sigma_{0}} \mathscr{A}_{\sigma}(\tau, \omega) \text { is precompact in } X .
$$

Then, for P-a.e. $\tau \in \mathbf{R}, \omega \in \Omega$,

$$
\operatorname{dist}\left(\mathscr{A}_{\sigma}(\tau, \omega), \mathscr{A}_{0}(\tau, \omega)\right) \longrightarrow 0, \quad \text { as } \sigma \longrightarrow 0
$$

To investigate the random dynamics of stochastic PDEs, we convert the stochastic evolutionary system to a system of pathwise PDEs with the random parameter. Let

$$
\begin{aligned}
& U\left(t, \tau, \omega, U_{\tau}\right)=e^{-\sigma z\left(\theta_{t} \omega\right)} u\left(t, \tau, \omega, U_{\tau}\right), \\
& V\left(t, \tau, \omega, V_{\tau}\right)=e^{-\sigma z\left(\theta_{t} \omega\right)} v\left(t, \tau, \omega, v_{\tau}\right)
\end{aligned}
$$

where $z\left(\theta_{t} \omega\right)$ is the Ornstein-Uhlenbeck process. Then

$$
\begin{aligned}
& d U=e^{-\sigma z\left(\theta_{t} \omega\right)} d u-\sigma e^{-\sigma z\left(\theta_{t} \omega\right)} u \circ d z\left(\theta_{t} \omega\right), \\
& d V=e^{-\sigma z\left(\theta_{t} \omega\right)} d v-\sigma e^{-\sigma z\left(\theta_{t} \omega\right)} v \circ d z\left(\theta_{t} \omega\right) .
\end{aligned}
$$

So we can consider the following equations with random coefficients, but without white noise:

$$
\begin{aligned}
\frac{d U}{d t}= & d_{1} \Delta U+e^{-\sigma z\left(\theta_{t} \omega\right)} \rho-a U+e^{2 \sigma z\left(\theta_{t} \omega\right)} U^{2} V \\
& -G e^{2 \sigma z\left(\theta_{t} \omega\right)} U^{3}+e^{-\sigma z\left(\theta_{t} \omega\right)} f_{1}(t, x) \\
& +\sigma U z\left(\theta_{t} \omega\right)
\end{aligned}
$$

$$
J\left(g, \theta_{t} \omega\right)=\left(\begin{array}{l}
e^{-\sigma z\left(\theta_{t} \omega\right)} \rho-a U+e^{2 \sigma z\left(\theta_{t} \omega\right)} U^{2} V-G e^{2 \sigma z\left(\theta_{t} \omega\right)} U^{3}+e^{-\sigma z\left(\theta_{t} \omega\right)} f_{1}(t, x)+\sigma U z\left(\theta_{t} \omega\right) \\
e^{-\sigma z\left(\theta_{t} \omega\right)} \beta-b V-e^{2 \sigma z\left(\theta_{t} \omega\right)} U^{2} V+G e^{2 \sigma z\left(\theta_{t} \omega\right)} U^{3}+e^{-\sigma z\left(\theta_{t} \omega\right)} f_{2}(t, x)+\sigma V z\left(\theta_{t} \omega\right)
\end{array}\right)
$$

for any $t \geq \tau$ with initial data

$$
\begin{aligned}
g_{\tau}(x) & =\left(U_{\tau}(x), V_{\tau}(x)\right)^{\mathbb{T}} \\
& =\left(e^{-\sigma z\left(\theta_{\tau} \omega\right)} u_{\tau}, e^{-\sigma z\left(\theta_{\tau} \omega\right)} v_{\tau}\right)^{\mathbb{T}} .
\end{aligned}
$$

By the Galerkin method, we can prove the existence and uniqueness of the weak solution, which is continuously depending on the initial data and

$$
g\left(t, \tau, \omega, g_{\tau}\right) \in C([\tau,+\infty) ; H) \cap L^{2}([\tau,+\infty) ; E) .
$$

This is similar to the autonomous case studied in [4]. Define the continuous random dynamical system $\varphi$ associated with the problem (23)-(24) as follows:

$$
\begin{aligned}
& \varphi: \mathbf{R}^{+} \times \mathbf{R} \times \Omega \times H \rightarrow H, \\
& \begin{array}{l}
\varphi\left(t, \tau, \omega, g_{\tau}\right)=g\left(t+\tau, \tau, \theta_{-\tau} \omega, g_{\tau}\right) \\
\quad=\left(U\left(t+\tau, \tau, \theta_{-\tau} \omega, U_{\tau}\right), V\left(t+\tau, \tau, \theta_{-\tau} \omega, V_{\tau}\right)\right)
\end{array}
\end{aligned}
$$

$$
\begin{aligned}
\frac{d V}{d t}= & d_{2} \Delta V+e^{-\sigma z\left(\theta_{t} \omega\right)} \beta-b V-e^{2 \sigma z\left(\theta_{t} \omega\right)} U^{2} V \\
& +G e^{2 \sigma z\left(\theta_{t} \omega\right)} U^{3}+e^{-\sigma z\left(\theta_{t} \omega\right)} f_{2}(t, x) \\
& +\sigma V z\left(\theta_{t} \omega\right),
\end{aligned}
$$

for $x \in \Gamma$ and $t \geq \tau$, with the homogeneous Dirichlet boundary conditions

$$
U(t, x)=V(t, x)=0, \quad t \geq \tau \in \mathbf{R}, x \in \partial \Gamma,
$$

and the initial value conditions

$$
\begin{aligned}
& U(\tau, x)=U_{\tau}(x)=e^{-\sigma z\left(\theta_{t} \omega\right)} u_{\tau}(x), \\
& V(\tau, x)=V_{\tau}(x)=e^{-\sigma z\left(\theta_{t} \omega\right)} v_{\tau}(x) .
\end{aligned}
$$

For every $\omega \in \Omega, \tau \in \mathbf{R}$, the initial-boundary value problem (19)-(22) is formulated into the following reversible Selkov equation:

$$
\begin{gathered}
\frac{d g}{d t}=A g+J(g)+f(t, x), \\
g(\tau, x)=g_{\tau}(x)=\left(U_{\tau}(x), V_{\tau}(x)\right)^{\mathbb{T}},
\end{gathered}
$$

where $g\left(t, \tau, \omega, g_{\tau}\right)=\left(U\left(t, \tau, \omega, U_{\tau}\right), V\left(t, \tau, \omega, V_{\tau}\right)\right)^{\mathbb{T}}$, and

$$
A=\left(\begin{array}{cc}
d_{1} \triangle & 0 \\
0 & d_{2} \triangle
\end{array}\right)
$$

and for all $\left(t, \tau, \omega, g_{\tau}\right) \in \mathbf{R}^{+} \times \mathbf{R} \times \Omega \times H$. The continuous dynamical system $\varphi$ and a continuous random dynamical system associated with the solutions of (1)-(4) are equivalent. Thus, we only discuss the dynamical system $\varphi$ induced by (19)-(22).

Assume that

$$
\begin{aligned}
X & =\left\{f \text { is measurable: } \int _ { - \infty } ^ { 0 } e ^ { ( \gamma / 2 ) s } \left(\|f(s+\tau, \cdot)\|_{L^{p}}^{p} \mathrm{~d} s\right.\right. \\
& <+\infty, p=2,4,6\} .
\end{aligned}
$$

Sometimes, we also assume that $f_{1}$ and $f_{2}$ are tempered in the following sense. For every $c>0$,

$$
\begin{aligned}
& \lim _{\lambda \longrightarrow-\infty} e^{c \lambda} \int_{-\infty}^{0} e^{(\gamma / 2) s}\left(\left\|f_{1}(s-\lambda, \cdot)\right\|_{L^{p}}^{p}\right. \\
& \left.\quad+\left\|f_{2}(s-\lambda, \cdot)\right\|_{L^{p}}^{p}\right) \mathrm{d} s=0, \quad p=2,4,6 .
\end{aligned}
$$




\section{Existence of Random Attractors}

In this section, we derive uniform estimates of solution for the stochastic reversible Selkov equations; these estimates are necessary for proving the existence of bounded absorbing sets and the asymptotic compactness of the random dynamical system. We will use $\mathscr{D}$ to denote the collection of all tempered random subsets of $H$ from now on.

For brevity, we write $U\left(t, \tau, \omega, U_{\tau}\right), V\left(t, \tau, \omega, V_{\tau}\right)$ as $U(t, \omega), V(t, \omega)$ or simply as $U, V$; similarly, we write weak solution $g\left(t, \tau, \omega, g_{\tau}\right)$ as $g(t, \omega)$ or $g$.

Lemma 4. Suppose that $f_{i}(t, x) \in X, i=1,2$. Then, for every $\tau \in \mathbf{R}, \omega \in \Omega$, and $B=\{B(\tau, \omega): \tau \in \mathbf{R}, \omega \in \Omega\} \in \mathscr{D}$, there exist a number $T_{0}(\tau, \omega, B, \sigma) \geq 0$ and a tempered random variable $M_{0}(\tau, \omega)$

$$
\begin{aligned}
& M_{0}(\tau, \omega) \\
& =1+C_{1} \int_{-\infty}^{0} e^{-2 \sigma z\left(\theta_{s} \omega\right)-\int_{0}^{s} 2 \sigma z\left(\theta_{r} \omega\right) d r+\gamma s} d s \\
& \quad+\frac{2 G}{a} \int_{-\infty}^{0} e^{-2 \sigma z\left(\theta_{s} \omega\right)-\int_{0}^{s} 2 \sigma z\left(\theta_{r} \omega\right) d r+\gamma s}\left\|f_{1}(s+\tau)\right\|^{2} d s \\
& \quad+\frac{2}{b} \int_{-\infty}^{0} e^{-2 \sigma z\left(\theta_{s} \omega\right)-\int_{0}^{s} 2 \sigma z\left(\theta_{r} \omega\right) d r+\gamma s}\left\|f_{2}(s+\tau)\right\|^{2} d s,
\end{aligned}
$$

such that for all $t \geq T_{0}$, the solution $g$ of (19)-(22) with $g_{\tau-t} \in$ $B\left(\tau-t, \theta_{-t} \omega\right)$ satisfies

$$
\begin{aligned}
\left\|g\left(\tau, \tau-t, \theta_{-\tau} \omega, g_{\tau-t}\right)\right\| \leq M_{0}(\tau, \omega) & \\
\forall t & \geq T_{0}(\tau, \omega, B, \sigma) .
\end{aligned}
$$

Proof. Multiplying (19) by $G U$ and (20) by $V$, we get

$$
\begin{aligned}
\frac{1}{2} \frac{\mathrm{d}}{\mathrm{d} t} G\|U\|^{2}+G d_{1}\|\nabla U\|^{2} \\
=e^{-\sigma z\left(\theta_{t} \omega\right)} \int_{\Gamma} G U \rho \mathrm{d} x-a G\|U\|^{2} \\
\quad+e^{2 \sigma z\left(\theta_{t} \omega\right)} \int_{\Gamma} G U^{3} V \mathrm{~d} x-e^{2 \sigma z\left(\theta_{t} \omega\right)} \int_{\Gamma} G^{2} U^{4} \mathrm{~d} x \\
\quad+\int_{\Gamma} e^{-\sigma z\left(\theta_{t} \omega\right)} f_{1}(t, x) G U d x \\
\quad+\sigma z\left(\theta_{t} \omega\right) G\|U\|^{2},
\end{aligned}
$$

and

$$
\begin{aligned}
\frac{1}{2} \frac{\mathrm{d}}{\mathrm{d} t} & \|V\|^{2}+d_{2}\|\nabla V\|^{2} \\
= & e^{-\sigma z\left(\theta_{t} \omega\right)} \int_{\Gamma} V \beta \mathrm{d} x-b\|V\|^{2} \\
& -e^{2 \sigma z\left(\theta_{t} \omega\right)} \int_{\Gamma} U^{2} V^{2} \mathrm{~d} x+e^{2 \sigma z\left(\theta_{t} \omega\right)} \int_{\Gamma} G U^{3} V \mathrm{~d} x \\
& +\int_{\Gamma} e^{-\sigma z\left(\theta_{t} \omega\right)} f_{2}(t, x) V d x+\sigma z\left(\theta_{t} \omega\right)\|V\|^{2} .
\end{aligned}
$$

Adding up the above two equations, we obtain

$$
\begin{aligned}
\frac{\mathrm{d}}{\mathrm{d} t}(G & \left.\|U\|^{2}+\|V\|^{2}\right)+2 G d_{1}\|\nabla U\|^{2}+2 d_{2}\|\nabla V\|^{2} \\
& +2\left(a G\|U\|^{2}+b\|V\|^{2}\right) \\
& -2 \sigma z\left(\theta_{t} \omega\right)\left(G\|U\|^{2}+\|V\|^{2}\right) \\
= & 2 e^{-\sigma z\left(\theta_{t} \omega\right)} \int_{\Gamma}\left(G U f_{1}(t, x)+V f_{2}(t, x)\right) d x \\
& +2 e^{-\sigma z\left(\theta_{t} \omega\right)} \int_{\Gamma}(G U \rho+V \beta) \mathrm{d} x \\
& -2 e^{2 \sigma z\left(\theta_{t} \omega\right)} \int_{\Gamma}\left(G U^{2}-U V\right)^{2} \mathrm{~d} x .
\end{aligned}
$$

By the $\epsilon$-Young inequality, we have

$$
\begin{aligned}
e^{-\sigma z\left(\theta_{t} \omega\right)} \int_{\Gamma}(G U \rho+V \beta) \mathrm{d} x \\
\leq \frac{a G}{4}\|U\|^{2}+\frac{b}{4}\|V\|^{2}+\frac{e^{-2 \sigma z\left(\theta_{t} \omega\right)} \rho^{2}|\Gamma| G}{a} \\
\quad+\frac{e^{-2 \sigma z\left(\theta_{t} \omega\right)} \beta^{2}|\Gamma|}{b}, \\
e^{-\sigma z\left(\theta_{t} \omega\right)} \int_{\Gamma}\left(G U f_{1}(t, x)+V f_{2}(t, x)\right) d x \\
\leq \frac{a G}{4}\|U\|^{2}+\frac{b}{4}\|V\|^{2}+\frac{e^{-2 \sigma z\left(\theta_{t} \omega\right)} G}{a}\left\|f_{1}\right\|^{2} \\
+\frac{e^{-2 \sigma z\left(\theta_{t} \omega\right)}}{b}\left\|f_{2}\right\|^{2} .
\end{aligned}
$$

It follows that

$$
\begin{aligned}
\frac{\mathrm{d}}{\mathrm{d} t}(G & \left.\|U\|^{2}+\|V\|^{2}\right)+2 G d_{1}\|\nabla U\|^{2}+2 d_{2}\|\nabla V\|^{2} \\
& +2\left(a G\|U\|^{2}+b\|V\|^{2}\right) \\
& -2 \sigma z\left(\theta_{t} \omega\right)\left(G\|U\|^{2}+\|V\|^{2}\right) \\
\leq & a G\|U\|^{2}+b\|V\|^{2} \\
& +e^{-2 \sigma z\left(\theta_{t} \omega\right)}\left(\frac{2 \rho^{2}|\Gamma| G}{a}+\frac{2 \beta^{2}|\Gamma|}{b}\right) \\
& +e^{-2 \sigma z\left(\theta_{t} \omega\right)}\left(\frac{2 G}{a}\left\|f_{1}\right\|^{2}+\frac{2}{b}\left\|f_{2}\right\|^{2}\right)
\end{aligned}
$$

Let $\gamma=\min \{a, b\}, C_{1}=2 \rho^{2}|\Gamma| G / a+2 \beta^{2}|\Gamma| / b$. It is easy to see that

$$
\begin{aligned}
& \frac{\mathrm{d}}{\mathrm{d} t}\left(G\|U\|^{2}+\|V\|^{2}\right) \\
& \quad+\left(\gamma-2 \sigma z\left(\theta_{t} \omega\right)\right)\left(G\|U\|^{2}+\|V\|^{2}\right)
\end{aligned}
$$




$$
\begin{aligned}
& +2 G d_{1}\|\nabla U\|^{2}+2 d_{2}\|\nabla V\|^{2} \\
\leq & C_{1} e^{-2 \sigma z\left(\theta_{t} \omega\right)}+e^{-2 \sigma z\left(\theta_{t} \omega\right)}\left(\frac{2 G}{a}\left\|f_{1}\right\|^{2}+\frac{2}{b}\left\|f_{2}\right\|^{2}\right) .
\end{aligned}
$$

By Gronwall's lemma on the interval $[\tau-t, r](r \geq \tau-t)$ for above inequality and substituting $\omega$ by $\theta_{-\tau} \omega$, we can deduce

$$
\begin{aligned}
& G\left\|U\left(r, \tau-t, \theta_{-\tau} \omega, U_{\tau-t}\right)\right\|^{2}+\| V\left(r, \tau-t, \theta_{-\tau}(\omega),\right. \\
& \left.V_{\tau-t}\right) \|^{2} \leq e^{\int_{-t}^{r-\tau} 2 \sigma z\left(\theta_{s} \omega\right) \mathrm{d} s-\gamma(r+t-\tau)}\left(G\left\|U_{\tau-t}\right\|^{2}\right. \\
& \left.+\left\|V_{\tau-t}\right\|^{2}\right) \\
& +C_{1} \int_{-t}^{r-\tau} e^{-2 \sigma z\left(\theta_{s} \omega\right)+\int_{s}^{r-\tau} 2 \sigma z\left(\theta_{r} \omega\right) \mathrm{d} r+\gamma(s+\tau-r)} \mathrm{d} s+\frac{2 G}{a} \\
& \quad \cdot \int_{-t}^{r-\tau} e^{-2 \sigma z\left(\theta_{s} \omega\right)+\int_{s}^{r-\tau} 2 \sigma z\left(\theta_{r} \omega\right) \mathrm{d} r+\gamma(s+\tau-r)} \| f_{1}(s \\
& +\tau) \|^{2} \mathrm{~d} s+\frac{2}{b} \\
& \quad \cdot \int_{-t}^{r-\tau} e^{-2 \sigma z\left(\theta_{s} \omega\right)+\int_{s}^{r-\tau} 2 \sigma z\left(\theta_{r} \omega\right) \mathrm{d} r+\gamma(s+\tau-r)} \| f_{2}(s \\
& +\tau) \|^{2} \mathrm{~d} s .
\end{aligned}
$$

Note that

$$
\begin{aligned}
& \frac{2 G}{a} \int_{-t}^{r-\tau} e^{-2 \sigma z\left(\theta_{s} \omega\right)+\int_{s}^{r-\tau} 2 \sigma z\left(\theta_{r} \omega\right) \mathrm{d} r+\gamma(s+\tau-r)}\left\|f_{1}(s+\tau)\right\|^{2} \mathrm{~d} s \\
& =\frac{2 G}{a} e^{\gamma(\tau-r)} e^{-\int_{r-\tau}^{0} 2 \sigma z\left(\theta_{r} \omega\right) \mathrm{d} r} \int_{-t}^{r-\tau} e^{\gamma s} e^{-\int_{0}^{s} 2 \sigma z\left(\theta_{r} \omega\right) \mathrm{d} r-2 \sigma z\left(\theta_{s} \omega\right)}\left\|f_{1}(s+\tau)\right\|^{2} \mathrm{~d} s .
\end{aligned}
$$

By (11) and (12), we find that there exists a $T_{1}=$ $T_{1}\left(\tau, \omega, B, \sigma_{0}\right)\left(0<\sigma<\sigma_{0}\right)$ such that for $\forall t \geq T_{1}>0$, we have

$$
\begin{array}{r}
\left|z\left(\theta_{-t} \omega\right)\right| \leq \frac{\gamma t}{8 \sigma_{0}} \\
\left|\int_{0}^{-t} z\left(\theta_{r} \omega\right) \mathrm{d} r\right| \leq \frac{\gamma t}{8 \sigma_{0}}
\end{array}
$$

We get for all $t \geq T_{1}$,

$$
\begin{gathered}
\int_{-t}^{-T_{1}} e^{\gamma s} e^{-\int_{0}^{s} 2 \sigma z\left(\theta_{r} \omega\right) \mathrm{d} r-2 \sigma z\left(\theta_{s} \omega\right)}\left\|f_{1}(s+\tau)\right\|^{2} \mathrm{~d} s \\
\leq \int_{-t}^{-T_{1}} e^{(\gamma / 2) s}\left\|f_{1}(s+\tau)\right\|^{2} \mathrm{~d} s .
\end{gathered}
$$

For the term on the right-hand side of (45), since $f_{1}, f_{2} \in X$, we have

$$
\int_{-t}^{-T_{1}} e^{(\gamma / 2) s}\left(\left\|f_{1}(s+\tau)\right\|^{2}+\left\|f_{2}(s+\tau)\right\|^{2}\right) \mathrm{d} s<+\infty .
$$

Thus, we get

$$
\begin{aligned}
& \frac{2 G}{a} \int_{-t}^{r-\tau} e^{-2 \sigma z\left(\theta_{s} \omega\right)-\int_{r-\tau}^{s} 2 \sigma z\left(\theta_{r} \omega\right) \mathrm{d} r+\gamma(s+\tau-r)} \| f_{1}(s \\
& \quad+\tau) \|^{2} \mathrm{~d} s \leq \frac{2 G}{a} \\
& \quad \cdot \int_{-\infty}^{r-\tau} e^{-2 \sigma z\left(\theta_{s} \omega\right)-\int_{r-\tau}^{s} 2 \sigma z\left(\theta_{r} \omega\right) \mathrm{d} r+\gamma(s+\tau-r)} \| f_{1}(s \\
& \quad+\tau) \|^{2} \mathrm{~d} s,
\end{aligned}
$$

where the integral is convergent due to (46). Similarly, we also obtain

$$
\begin{aligned}
& \frac{2}{b} \int_{-t}^{r-\tau} e^{-2 \sigma z\left(\theta_{s} \omega\right)-\int_{r-\tau}^{s} 2 \sigma z\left(\theta_{r} \omega\right) \mathrm{d} r+\gamma(s+\tau-r)}\left\|f_{2}(s+\tau)\right\|^{2} \mathrm{~d} s \\
& \quad \leq \frac{2}{b} \int_{-\infty}^{r-\tau} e^{-2 \sigma z\left(\theta_{s} \omega\right)-\int_{r-\tau}^{s} 2 \sigma z\left(\theta_{r} \omega\right) \mathrm{d} r+\gamma(s+\tau-r)} \| f_{2}(s \\
& \quad+\tau) \|^{2} \mathrm{~d} s .
\end{aligned}
$$

Thus, we yield

$$
\begin{aligned}
& G\left\|U\left(r, \tau-t, \theta_{-\tau} \omega, U_{\tau-t}\right)\right\|^{2} \\
& +\left\|V\left(r, \tau-t, \theta_{-\tau}(\omega), V_{\tau-t}\right)\right\|^{2} \\
& \leq e^{\int_{-t}^{r-\tau} 2 \sigma z\left(\theta_{s} \omega\right) \mathrm{d} s-\gamma(r+t-\tau)}\left(G\left\|U_{\tau-t}\right\|^{2}+\left\|V_{\tau-t}\right\|^{2}\right) \\
& +C_{1} \int_{-t}^{r-\tau} e^{-2 \sigma z\left(\theta_{s} \omega\right)+\int_{s}^{r-\tau} 2 \sigma z\left(\theta_{r} \omega\right) \mathrm{d} r+\gamma(s+\tau-r)} \mathrm{d} s \\
& +\frac{2 G}{a} \int_{-\infty}^{r-\tau} e^{-2 \sigma z\left(\theta_{s} \omega\right)+\int_{s}^{r-\tau} 2 \sigma z\left(\theta_{r} \omega\right) \mathrm{d} r+\gamma(s+\tau-r)}\left\|f_{1}(s+\tau)\right\|^{2} \mathrm{~d} s \\
& +\frac{2}{b} \int_{-\infty}^{r-\tau} e^{-2 \sigma z\left(\theta_{s} \omega\right)+\int_{s}^{r-\tau} 2 \sigma z\left(\theta_{r} \omega\right) \mathrm{d} r+\gamma(s+\tau-r)}\left\|f_{2}(s+\tau)\right\|^{2} \mathrm{~d} s .
\end{aligned}
$$

By (49), we get that

$$
\begin{aligned}
& G\left\|U\left(\tau, \tau-t, \theta_{-\tau} \omega, U_{\tau-t}\right)\right\|^{2} \\
& +\left\|V\left(\tau, \tau-t, \theta_{-\tau} \omega, V_{\tau-t}\right)\right\|^{2} \\
& \leq e^{\int_{-t}^{0} 2 \sigma z\left(\theta_{s} \omega\right) \mathrm{d} s-\gamma t}\left(G\left\|U_{\tau-t}\right\|^{2}+\left\|V_{\tau-t}\right\|^{2}\right) \\
& \quad+C_{1} \int_{-\infty}^{0} e^{-2 \sigma z\left(\theta_{s} \omega\right)+\int_{s}^{0} 2 \sigma z\left(\theta_{r} \omega\right) \mathrm{d} r+\gamma s} \mathrm{~d} s \\
& \quad+\frac{2 G}{a} \int_{-\infty}^{0} e^{-2 \sigma z\left(\theta_{s} \omega\right)+\int_{s}^{0} 2 \sigma z\left(\theta_{r} \omega\right) \mathrm{d} r+\gamma s}\left\|f_{1}(s+\tau)\right\|^{2} \mathrm{~d} s \\
& \quad+\frac{2}{b} \int_{-\infty}^{0} e^{-2 \sigma z\left(\theta_{s} \omega\right)+\int_{s}^{0} 2 \sigma z\left(\theta_{r} \omega\right) \mathrm{d} r+\gamma s}\left\|f_{2}(s+\tau)\right\|^{2} \mathrm{~d} s .
\end{aligned}
$$

According to the properties of the Ornstein-Uhlenbeck process,

$$
\int_{-\infty}^{0} e^{-2 \sigma z\left(\theta_{s} \omega\right)+\int_{s}^{0} 2 \sigma z\left(\theta_{r} \omega\right) \mathrm{d} r+\gamma s} \mathrm{~d} s<+\infty
$$


Noticing that $\{B(\tau, \omega): \tau \in \mathbf{R}, \omega \in \Omega\} \in \mathscr{D}$ is tempered, then for any $\left(U_{\tau-t}, V_{\tau-t}\right) \in B\left(\tau-t, \theta_{-t} \omega\right)$, we have

$$
\lim _{t \longrightarrow+\infty} e^{\int_{-t}^{0}\left(2 \sigma z\left(\theta_{s} \omega\right)-\gamma\right) \mathrm{d} s}\left(G\left\|U_{\tau-t}\right\|^{2}+\left\|V_{\tau-t}\right\|^{2}\right)=0 .
$$

Then, $M_{0}(\tau, \omega)$ is tempered. By (49), the proof is completed.

Lemma 5. Suppose that $f_{i}(t, x) \in X, i=1,2$. Then, for every $\tau \in \mathbf{R}, \omega \in \Omega$, and $B=\{B(\tau, \omega): \tau \in \mathbf{R}, \omega \in \Omega\} \in \mathscr{D}$, there exist a number $T_{2}(\tau, \omega, B, \sigma) \geq 0$ and tempered random variables $M_{1}(\tau, \omega), M_{2}(\tau, \omega)$

$$
\begin{aligned}
& M_{1}(\tau, \omega)=1 \\
& +\frac{C_{1}}{2} \\
& \cdot e^{\int_{-1}^{0}\left|2 \sigma z\left(\theta_{s} \omega\right)\right| d s+\gamma} \int_{-\infty}^{0} e^{-2 \sigma z\left(\theta_{s} \omega\right)+\int_{s}^{0} 2 \sigma z\left(\theta_{r} \omega\right) d r+\gamma s} d s \\
& +\frac{G}{a} e^{\int_{-1}^{0}\left|2 \sigma z\left(\theta_{s} \omega\right)\right| d s+\gamma} \int_{-\infty}^{0} e^{-2 \sigma z\left(\theta_{s} \omega\right)+\int_{s}^{0} 2 \sigma z\left(\theta_{r} \omega\right) d r+\gamma s} \| f_{1}(s \\
& +\tau) \|^{2} d s \\
& +\frac{1}{b} e^{\int_{-1}^{0}\left|2 \sigma z\left(\theta_{s} \omega\right)\right| d s+\gamma} \int_{-\infty}^{0} e^{-2 \sigma z\left(\theta_{s} \omega\right)+\int_{s}^{0} 2 \sigma z\left(\theta_{r} \omega\right) d r+\gamma s} \| f_{2}(s \\
& +\tau) \|^{2} d s, \\
& M_{2}(\tau, \omega)=1 \\
& +\frac{C_{1}}{2} \\
& +e^{\int_{-2}^{-1}\left|2 \sigma z\left(\theta_{s} \omega\right)\right| d s+(\gamma+1)} \int_{-\infty}^{-1} e^{-2 \sigma z\left(\theta_{s} \omega\right)+\int_{s}^{-1} 2 \sigma z\left(\theta_{r} \omega\right) d r+\gamma(s+1)} d s \\
& +\frac{G}{a} e^{\int_{-2}^{-1}\left|2 \sigma z\left(\theta_{s} \omega\right)\right| d s+(\gamma+1)} \int_{-\infty}^{-1} e^{-2 \sigma z\left(\theta_{s} \omega\right)+\int_{s}^{-1} 2 \sigma z\left(\theta_{r} \omega\right) d r+\gamma(s+1)} \| f_{1}(s \\
& +\tau) \|^{2} d s \\
& +\frac{1}{b} e^{\int_{-2}^{-1}\left|2 \sigma z\left(\theta_{s} \omega\right)\right| d s+(\gamma+1)} \int_{-\infty}^{-1} e^{-2 \sigma z\left(\theta_{s} \omega\right)+\int_{s}^{-1} 2 \sigma z\left(\theta_{r} \omega\right) d r+\gamma(s+1)} \| f_{2}(s \\
& +\tau) \|^{2} d s .
\end{aligned}
$$

such that for all $t \geq T_{2}$, the solution $g$ of (19)-(22) with $g_{\tau-t} \in$ $B\left(\tau-t, \theta_{-t} \omega\right)$ satisfies

$$
\begin{aligned}
& \int_{\tau-1}^{\tau}\left\|\nabla U\left(s, \tau-t, \theta_{-\tau} \omega, U_{\tau-t}\right)\right\|^{2} d s \\
& \quad+\int_{\tau-1}^{\tau}\left\|\nabla V\left(s, \tau-t, \theta_{-\tau} \omega, V_{\tau-t}\right)\right\|^{2} d s \leq M_{1}(\tau, \omega), \\
& \int_{\tau-2}^{\tau-1}\left\|\nabla U\left(s, \tau-t, \theta_{-\tau} \omega, U_{\tau-t}\right)\right\|^{2} d s \\
& \quad+\int_{\tau-2}^{\tau-1}\left\|\nabla V\left(s, \tau-t, \theta_{-\tau} \omega, V_{\tau-t}\right)\right\|^{2} d s \leq M_{2}(\tau, \omega),
\end{aligned}
$$

Proof. Substituting $r$ for $\tau-1$ in (41), for $t \geq 1$, we yield

$$
\begin{aligned}
G & \left\|U\left(\tau-1, \tau-t, \theta_{-\tau} \omega, U_{\tau-t}\right)\right\|^{2} \\
& +\left\|V\left(\tau-1, \tau-t, \theta_{-\tau}(\omega), V_{\tau-t}\right)\right\|^{2} \\
& \leq e^{\int_{-t}^{-1} 2 \sigma z\left(\theta_{s} \omega\right) \mathrm{d} s-\gamma(t-1)}\left(G\left\|U_{\tau-t}\right\|^{2}+\left\|V_{\tau-t}\right\|^{2}\right) \\
& +C_{1} \int_{-t}^{-1} e^{-2 \sigma z\left(\theta_{s} \omega\right)+\int_{s}^{-1} 2 \sigma z\left(\theta_{r} \omega\right) \mathrm{d} r+\gamma(1+s)} \mathrm{d} s+\frac{2 G}{a} \\
& \cdot \int_{-t}^{-1} e^{-2 \sigma z\left(\theta_{s} \omega\right)+\int_{s}^{-1} 2 \sigma z\left(\theta_{r} \omega\right) \mathrm{d} r+\gamma(1+s)}\left\|f_{1}(s+\tau)\right\|^{2} \mathrm{~d} s \\
& +\frac{2}{b} \\
& \cdot \int_{-t}^{-1} e^{-2 \sigma z\left(\theta_{s} \omega\right)+\int_{s}^{-1} 2 \sigma z\left(\theta_{r} \omega\right) \mathrm{d} r+\gamma(1+s)}\left\|f_{2}(s+\tau)\right\|^{2} \mathrm{~d} s .
\end{aligned}
$$

Applying Gronwall's lemma to (40) over $[\tau-1, \tau]$ and by (55), we obtain

$$
\begin{aligned}
& d_{1} G \int_{\tau-1}^{\tau} e^{\int_{s}^{\tau} 2 \sigma z\left(\theta_{s-\tau} \omega\right) \mathrm{d} s-\gamma(\tau-s)} \| \nabla U(s, \tau \\
& \left.-t, \theta_{-\tau} \omega, U_{\tau-t}\right) \|^{2} \mathrm{~d} s \\
& +d_{2} \int_{\tau-1}^{\tau} e^{\int_{s}^{\tau} 2 \sigma z\left(\theta_{s-\tau} \omega\right) \mathrm{d} s-\gamma(\tau-s)} \| \nabla V(s, \tau \\
& \left.-t, \theta_{-\tau} \omega, V_{\tau-t}\right) \|^{2} \mathrm{~d} s \leq \frac{1}{2} \\
& \cdot e^{\int_{-t}^{0} 2 \sigma z\left(\theta_{s} \omega\right) \mathrm{d} s-\gamma t}\left(G\left\|U_{\tau-t}\left(\theta_{-\tau} \omega\right)\right\|^{2}\right. \\
& \left.+\left\|V_{\tau-t}\left(\theta_{-\tau} \omega\right)\right\|^{2}\right)+\frac{C_{1}}{2} \\
& \cdot \int_{-\infty}^{0} e^{-2 \sigma z\left(\theta_{s} \omega\right)+\int_{s}^{0} 2 \sigma z\left(\theta_{r} \omega\right) \mathrm{d} r+\gamma s} \mathrm{~d} s+\frac{G}{a} \\
& \quad \cdot \int_{-\infty}^{0} e^{-2 \sigma z\left(\theta_{s} \omega\right)+\int_{s}^{0} 2 \sigma z\left(\theta_{r} \omega\right) \mathrm{d} r+\gamma s}\left\|f_{1}(s+\tau)\right\|^{2} \mathrm{~d} s \\
& +\frac{1}{b} \int_{-\infty}^{0} e^{-2 \sigma z\left(\theta_{s} \omega\right)+\int_{s}^{0} 2 \sigma z\left(\theta_{r} \omega\right) \mathrm{d} r+\gamma s}\left\|f_{2}(s+\tau)\right\|^{2} \mathrm{~d} s
\end{aligned}
$$

It is easy to show that

$$
\begin{aligned}
& \int_{\tau-1}^{\tau} e^{\int_{s}^{\tau} 2 \sigma z\left(\theta_{s-\tau} \omega\right) \mathrm{d} s-\gamma(\tau-s)} \| \nabla U(s, \tau \\
& \left.-t, \theta_{-\tau} \omega, U_{\tau-t}\right) \|^{2} \mathrm{~d} s \\
& \geq e^{-\int_{-1}^{0}\left|2 \sigma z\left(\theta_{s} \omega\right)\right| \mathrm{d} s-\gamma} \int_{\tau-1}^{\tau} \| \nabla U(s, \tau \\
& \left.-t, \theta_{-\tau} \omega, U_{\tau-t}\right) \|^{2} \mathrm{~d} s,
\end{aligned}
$$


and

$$
\begin{aligned}
& \int_{\tau-1}^{\tau} e^{\int_{s}^{\tau} 2 \sigma z\left(\theta_{s-\tau} \omega\right) \mathrm{d} s-\gamma(\tau-s)} \| \nabla V(s, \tau \\
& \left.-t, \theta_{-\tau} \omega, V_{\tau-t}\right) \|^{2} \mathrm{~d} s \\
& \geq e^{-\int_{-1}^{0}\left|2 \sigma z\left(\theta_{s} \omega\right)\right| \mathrm{d} s-\gamma} \int_{\tau-1}^{\tau} \| \nabla V(s, \tau \\
& \left.-t, \theta_{-\tau} \omega, V_{\tau-t}\right) \|^{2} \mathrm{~d} s .
\end{aligned}
$$

Now let $d=\min \left\{d_{1}, d_{2}, d_{1} G\right\}$. According to (56), (57), and (58), there exists a $T_{3}(\tau, \omega, B, \sigma)$ such that for all $t \geq$ $T_{3}(\tau, \omega, B, \sigma)$, we can get

$$
\begin{aligned}
& \int_{\tau-1}^{\tau}\left\|\nabla U\left(s, \tau-t, \theta_{-\tau} \omega, U_{\tau-t}\right)\right\|^{2} \mathrm{~d} s \\
& +\int_{\tau-1}^{\tau} \| \nabla V(s, \tau \\
& \left.-t, \theta_{-\tau} \omega, V_{\tau-t}\right) \|^{2} \mathrm{~d} s \\
& \leq \frac{1}{d} e^{\int_{-1-1}^{0} 2 \sigma z z\left(\theta_{s} \omega\right) \mid d s+\gamma}\left[\int_{\tau-1}^{\tau} e^{\int_{s}^{\tau} \tau z z\left(\theta_{-\tau}-\omega\right) d s-\gamma(\tau-s)} \| \nabla U(s, \tau\right. \\
& \left.-t, \theta_{-\tau} \omega, U_{\tau-t}\right)\left\|^{2} \mathrm{~d} s+\int_{\tau-1}^{\tau} e^{\int_{s}^{\tau} 2 \sigma z z\left(\theta_{s-\tau} \omega\right) \mathrm{d} s-\gamma(\tau-s)}\right\| \nabla V(s, \tau \\
& \left.\left.-t, \theta_{-\tau} \omega, V_{\tau-t}\right) \|^{2} \mathrm{~d} s\right] \leq 1 \\
& +\frac{C_{1}}{2} \\
& \cdot e^{\int_{-1}^{0}\left|2 \sigma z\left(\theta_{s} \omega\right)\right| d \mathrm{~d} s+\gamma} \int_{-\infty}^{0} e^{-2 \sigma z\left(\theta_{s} \omega\right)+\int_{s}^{0} 2 \sigma z\left(\theta_{r} \omega\right) \mathrm{dr}+\gamma s} \mathrm{~d} s
\end{aligned}
$$

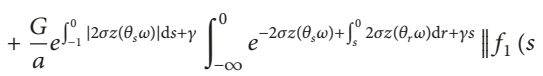

$$
\begin{aligned}
& +\tau) \|^{2} \mathrm{~d} s \\
& +\frac{1}{b} \\
& \cdot e^{\int_{-1}^{0}\left|2 \sigma z\left(\theta_{s} \omega\right)\right| \mathrm{d} s+\gamma} \int_{-\infty}^{0} e^{-2 \sigma z z\left(\theta_{s} \omega\right)+\int_{s}^{0} 2 \sigma z\left(\theta_{r} \omega\right) \mathrm{dr}+\gamma s}\left\|f_{2}(s+\tau)\right\|^{2} \mathrm{~d} s \doteq M_{1}(\tau, \omega) .
\end{aligned}
$$

Similarly, substituting $r$ for $\tau-2$ in (41), applying Gronwall's lemma to (40) over $[\tau-2, \tau-1]$, and combining the estimate (49), there exists a $T_{4}(\tau, \omega, B, \sigma)$ such that for all $t \geq T_{4}(\tau, \omega, B, \sigma)$, we get

$$
\begin{aligned}
& \int_{\tau-2}^{\tau-1}\left\|\nabla U\left(s, \tau-t, \theta_{-\tau} \omega, U_{\tau-t}\right)\right\|^{2} \mathrm{~d} s \\
& \quad+\int_{\tau-2}^{\tau-1} \| \nabla V(s, \\
& \left.\tau-t, \theta_{-\tau} \omega, V_{\tau-t}\right) \|^{2} \mathrm{~d} s \leq 1 \\
& \quad+\frac{C_{1}}{2} \\
& \quad \cdot e_{-2}^{\int_{-2}^{-1}\left|2 \sigma z\left(\theta_{s} \omega\right)\right| \mathrm{d} s+(\gamma+1)} \int_{-\infty}^{-1} e^{-2 \sigma z\left(\theta_{s} \omega\right)+\int_{s}^{-1} 2 \sigma z\left(\theta_{r} \omega\right) \mathrm{d} r+\gamma(s+1)} \mathrm{d} s \\
& \quad+\frac{G}{a} e^{\int_{-2}^{-1}\left|2 \sigma z\left(\theta_{s} \omega\right)\right| \mathrm{d} s+(\gamma+1)} \int_{-\infty}^{-1} e^{-2 \sigma z\left(\theta_{s} \omega\right)++_{s}^{-1} 2 \sigma z\left(\theta_{r} \omega\right) \mathrm{d} r+\gamma(s+1)} \| f_{1}(s \\
& \quad+\tau) \|^{2} \mathrm{~d} s
\end{aligned}
$$

$$
\begin{aligned}
& +\frac{1}{b} e^{\int_{-2}^{-1}\left|2 \sigma z\left(\theta_{s} \omega\right)\right| d s+(\gamma+1)} \int_{-\infty}^{-1} e^{-2 \sigma z\left(\theta_{s} \omega\right)+\int_{s}^{-1} 2 \sigma z\left(\theta_{r} \omega\right) \mathrm{d} r+\gamma(s+1)} \| f_{2}(s \\
& +\tau) \|^{2} \mathrm{~d} s \doteq M_{2}(\tau, \omega) .
\end{aligned}
$$

It is easy to check that $M_{1}(\tau, \omega)$ and $M_{2}(\tau, \omega)$ are tempered. Let $T_{2}=\max \left\{T_{3}, T_{4}\right\}$. This completes the proof.

Lemma 6. Let $f_{i}(t, x) \in X, i=1,2, q \in[\tau-1, \tau]$. If there exists a $t_{0} \in(\tau-t, q)$ such that $g\left(t_{0}, \tau-t, \theta_{-\tau} \omega, g_{\tau-t}\right) \in E$ with $\left\|g\left(t_{0}, \tau-t, \theta_{-\tau} \omega, g_{\tau-t}\right)\right\|_{E} \leq R(\tau, \omega)$, where $R(\tau, \omega)>0$ is any given random variable. Then for every $\tau \in \mathbf{R}, \omega \in \Omega$, and $B(\tau, \omega) \in \mathscr{D}$, there exist a number $T_{5}(\tau, \omega, B, \sigma) \geq 0$ and two tempered random variables $M_{3}(\tau, \omega)$ and $M_{4}(\tau, \omega)$

$$
\begin{aligned}
& M_{3}(\tau, \omega)=1+C_{2} \int_{-\infty}^{q-\tau} e^{-4 \sigma z\left(\theta_{s} \omega\right)+\int_{s}^{q-\tau} 4 \sigma z\left(\theta_{s} \omega\right) d s+\gamma(s-q+\tau)} d s
\end{aligned}
$$

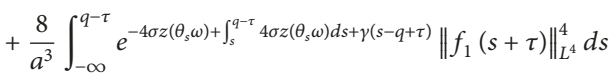

$$
\begin{aligned}
& +\frac{8}{b^{3} G^{3}} \int_{-\infty}^{q-\tau} e^{-4 \sigma z\left(\theta_{s}(\omega)+\int_{s}^{q-\tau} 4 \sigma z\left(\theta_{s}(\omega) d s+\gamma(s-q+\tau)\right.\right.}\left\|f_{2}(s+\tau)\right\|_{L^{4}}^{4} d s,
\end{aligned}
$$

and

$$
\begin{aligned}
& M_{4}(\tau, \omega)=1 \\
& +C_{3} \int_{-\infty}^{q-\tau} e^{-6 \sigma z\left(\theta_{s} \omega\right)+\int_{s}^{q-\tau} 6 \sigma z\left(\theta_{s} \omega\right) d s+\gamma(s-q+\tau)} d s+\frac{32}{a^{5}} \\
& \cdot \int_{-\infty}^{q-\tau} e^{-6 \sigma z\left(\theta_{s} \omega\right)+\int_{s}^{q-\tau} 6 \sigma z\left(\theta_{s} \omega\right) d s+\gamma(s-q+\tau)} \\
& \cdot\left\|f_{1}(s+\tau)\right\|_{L^{6}}^{6} d s+\frac{32}{b^{5} G^{5}} \\
& \cdot \int_{-\infty}^{q-\tau} e^{-6 \sigma z\left(\theta_{s} \omega\right)+\int_{s}^{0} 6 \sigma z\left(\theta_{s} \omega\right) d s+\gamma(s-q+\tau)} \\
& \cdot\left\|f_{2}(s+\tau)\right\|_{L^{6}}^{6} d s,
\end{aligned}
$$

such that for all $t \geq T_{5}$, the solution $g$ of (19)-(22) with $g_{\tau-t} \in$ $B\left(\tau-t, \theta_{-t} \omega\right)$ satisfies

$$
\begin{aligned}
& \left\|g\left(q, \tau-t, \theta_{-\tau} \omega, g_{\tau-t}\right)\right\|_{L^{4}}^{4} \leq M_{3}(\tau, \omega), \\
& \left\|g\left(q, \tau-t, \theta_{-\tau} \omega, g_{\tau-t}\right)\right\|_{L^{6}}^{6} \leq M_{4}(\tau, \omega) .
\end{aligned}
$$

Proof. Here we invoked Sobolev's embedding theorem to assert that $H_{0}^{1}(\Gamma) \hookrightarrow L^{6}(\Gamma) \hookrightarrow L^{4}(\Gamma)$ is a continuous embedding for $n \leq 3$. For $g\left(t_{0}, \tau-t, \theta_{-\tau} \omega, g_{\tau-t}\right) \in E$ with $\left\|g\left(t_{0}, \tau-t, \theta_{-\tau} \omega, g_{\tau-t}\right)\right\|_{E} \leq R(\tau, \omega)$, it follows that

$$
\begin{aligned}
& \left\|g\left(t_{0}, \tau-t, \theta_{-\tau} \omega, g_{\tau-t}\right)\right\|_{L^{6}} \leq \eta R(\tau, \omega), \\
& \left\|g\left(t_{0}, \tau-t, \theta_{-\tau} \omega, g_{\tau-t}\right)\right\|_{L^{4}} \leq \lambda R(\tau, \omega),
\end{aligned}
$$

where $\eta$ and $\lambda$ are positive constants. 
Let $\widetilde{U}=U, \widetilde{V}=V / G$. Then, the transformed system (19)(20) becomes a rescaled system

$$
\begin{aligned}
\frac{d \widetilde{U}}{d t}= & d_{1} \Delta \widetilde{U}+e^{-\sigma z\left(\theta_{s} \omega\right)} \rho-a \widetilde{U}+e^{2 \sigma z\left(\theta_{s} \omega\right)} G \widetilde{U}^{2} \widetilde{V} \\
& -G e^{2 \sigma z\left(\theta_{s} \omega\right)} \widetilde{U}^{3}+e^{-\sigma z\left(\theta_{s} \omega\right)} f_{1}(t, x) \\
& +\sigma \widetilde{U} z\left(\theta_{s} \omega\right), \\
\frac{d \widetilde{V}}{d t}= & d_{2} \Delta \widetilde{V}+\frac{e^{-\sigma z\left(\theta_{s} \omega\right)} \beta}{G}-b \widetilde{V}-e^{2 \sigma z\left(\theta_{s} \omega\right)} \widetilde{U}^{2} \widetilde{V} \\
& +e^{2 \sigma z\left(\theta_{s} \omega\right)} \widetilde{U}^{3}+\frac{e^{-\sigma z\left(\theta_{s} \omega\right)} f_{2}(t, x)}{G} \\
& +\sigma \widetilde{V} z\left(\theta_{s} \omega\right) .
\end{aligned}
$$

Multiplying (65) by $\widetilde{U}^{3}$, we obtain

$$
\begin{aligned}
& \frac{1}{4} \frac{\mathrm{d}}{\mathrm{d} t}\|\widetilde{U}\|_{L^{4}}^{4}+3 d_{1}\|\widetilde{U} \nabla \widetilde{U}\|^{2} \\
& =\int_{\Gamma} e^{-\sigma z\left(\theta_{s} \omega\right)}\left(\rho \widetilde{U}^{3}+f_{1} \widetilde{U}^{3}\right) d x-a\left\|\widetilde{U}^{4}\right\|_{L^{4}} \\
& \quad+\int_{\Gamma} e^{2 \sigma z\left(\theta_{s} \omega\right)}\left(G \widetilde{U}^{5} \widetilde{V}-G \widetilde{U}^{6}\right) \mathrm{d} x \\
& +\sigma z\left(\theta_{s} \omega\right)\|\widetilde{U}\|_{L^{4}}^{4} .
\end{aligned}
$$

Multiplying (66) by $G \widetilde{V}^{3}$, we get

$$
\begin{aligned}
& \frac{1}{4} \frac{\mathrm{d}}{\mathrm{d} t} G\|\widetilde{V}\|_{L^{4}}^{4}+3 G d_{2}\|\widetilde{V} \nabla \widetilde{V}\|^{2} \\
& =\int_{\Gamma} e^{-\sigma z\left(\theta_{s} \omega\right)}\left(\beta \widetilde{V}^{3}+f_{2} \widetilde{V}^{3}\right) \mathrm{d} x-b G\left\|\widetilde{V}^{4}\right\|_{L^{4}} \\
& \quad+\int_{\Gamma} e^{2 \sigma z\left(\theta_{s} \omega\right)}\left(-G \widetilde{U}^{2} \widetilde{V}^{4}+G \widetilde{U}^{3} \widetilde{V}^{3}\right) \mathrm{d} x \\
& \quad+\sigma G z\left(\theta_{s} \omega\right)\|\widetilde{V}\|_{L^{4}}^{4} .
\end{aligned}
$$

Summing up the resulting equalities, we have

$$
\begin{aligned}
& \frac{1}{4} \frac{\mathrm{d}}{\mathrm{d} t}\left(\|\widetilde{U}\|_{L^{4}}^{4}+G\|\widetilde{V}\|_{L^{4}}^{4}\right) \\
& \quad+3 d\left(\|\widetilde{U} \nabla \widetilde{U}\|^{2}+G\|\widetilde{V} \nabla \widetilde{V}\|^{2}\right) \\
& \quad \leq e^{-\sigma z\left(\theta_{s} \omega\right)} \int_{\Gamma}\left(\rho \widetilde{U}^{3}+\beta \widetilde{V}^{3}+f_{1} \widetilde{U}^{3}+f_{2} \widetilde{V}^{3}\right) \mathrm{d} x
\end{aligned}
$$

$$
\begin{aligned}
& -a\left\|\widetilde{U}^{4}\right\|_{L^{4}}-b G\left\|\widetilde{V}^{4}\right\|_{L^{4}} \\
& +e^{2 \sigma z\left(\theta_{s} \omega\right)} G \int_{\Gamma}\left(-\widetilde{U}^{6}+\widetilde{U}^{5} \widetilde{V}+\widetilde{U}^{3} \widetilde{V}^{3}-\widetilde{U}^{2} \widetilde{V}^{4}\right) \mathrm{d} x \\
& +\sigma z\left(\theta_{s} \omega\right)\left(\|\widetilde{U}\|_{L^{4}}^{4}+G\|\widetilde{V}\|_{L^{4}}^{4}\right) \\
& =e^{-\sigma z\left(\theta_{s} \omega\right)} \int_{\Gamma}\left(\rho \widetilde{U}^{3}+\beta \widetilde{V}^{3}+f_{1} \widetilde{U}^{3}+f_{2} \widetilde{V}^{3}\right) \mathrm{d} x \\
& -a\left\|\widetilde{U}^{4}\right\|_{L^{4}}-b G\left\|\widetilde{V}^{4}\right\|_{L^{4}} \\
& +e^{2 \sigma z\left(\theta_{s} \omega\right)} G \int_{\Gamma} \widetilde{U}^{2}\left(-\widetilde{U}^{4}+\widetilde{U}^{3} \widetilde{V}+\widetilde{U} \widetilde{V}^{3}-\widetilde{V}^{4}\right) \mathrm{d} x \\
& +\sigma z\left(\theta_{s} \omega\right)\left(\|\widetilde{U}\|_{L^{4}}^{4}+G\|\widetilde{V}\|_{L^{4}}^{4}\right) .
\end{aligned}
$$

Using the Young inequality, we have

$$
\begin{aligned}
& -\widetilde{U}^{4}+\widetilde{U}^{3} \widetilde{V}+\widetilde{U} \widetilde{V}^{3}-\widetilde{V}^{4} \\
& \quad \leq-\widetilde{U}^{4}+\left(\frac{3}{4} \widetilde{U}^{4}+\frac{1}{4} \widetilde{V}^{4}\right)+\left(\frac{1}{4} \widetilde{U}^{4}+\frac{3}{4} \widetilde{V}^{4}\right)-\widetilde{V}^{4} \\
& \quad \leq 0
\end{aligned}
$$

and

$$
\begin{aligned}
e^{-\sigma z\left(\theta_{s} \omega\right)} \int_{\Gamma}\left(\rho \widetilde{U}^{3}+\beta \widetilde{V}^{3}+f_{1} \widetilde{U}^{3}+f_{2} \widetilde{V}^{3}\right) \mathrm{d} x \\
\leq e^{-\sigma z\left(\theta_{s} \omega\right)}\left(\rho|\Gamma|^{1 / 4}\|\widetilde{U}\|_{L^{4}}^{3}+\beta|\Gamma|^{1 / 4}\|\widetilde{V}\|_{L^{4}}^{3}\right) \\
+e^{-\sigma z\left(\theta_{s} \omega\right)}\left(\left\|f_{1}\right\|_{L^{4}}\|\widetilde{U}\|_{L^{4}}^{3}+\left\|f_{2}\right\|_{L^{4}}\|\widetilde{V}\|_{L^{4}}^{3}\right) \\
\leq \frac{2}{a^{3}} e^{-4 \sigma z\left(\theta_{s} \omega\right)} \rho^{4}|\Gamma|+\frac{3 a}{8}\|\widetilde{U}\|_{L^{4}}^{4}+\frac{3 b G}{8}\|\widetilde{V}\|_{L^{4}}^{4} \\
+\frac{2}{b^{3} G^{3}} e^{-4 \sigma z\left(\theta_{s} \omega\right)} \beta^{4}|\Gamma|+\frac{2}{a^{3}} e^{-4 \sigma z\left(\theta_{s} \omega\right)}\left\|f_{1}\right\|_{L^{4}}^{4} \\
+\frac{3 a}{8}\|\widetilde{U}\|_{L^{4}}^{4}+\frac{2}{b^{3} G^{3}} e^{-4 \sigma z\left(\theta_{s} \omega\right)}\left\|f_{2}\right\|_{L^{4}}^{4} \\
+\frac{3 b G}{8}\|\widetilde{V}\|_{L^{4}}^{4} \\
+\frac{3}{4}\left(a\|\widetilde{U}\|_{L^{4}}^{4}+b G\|\widetilde{V}\|_{L^{4}}^{4}\right) \\
+e^{-4 \sigma z\left(\theta_{s} \omega\right)}\left(\frac{2 \rho^{4}|\Gamma|}{a^{3}}+\frac{2 \beta^{4}|\Gamma|}{b^{3} G^{3}}\right) \\
+4 \sigma z\left(\theta_{s} \omega\right)\left(\frac{2\left\|f_{1}\right\|_{L^{4}}^{4}}{a^{3}}+\frac{2\left\|f_{2}\right\|_{L^{4}}^{4}}{b^{3} G^{3}}\right) .
\end{aligned}
$$


Recalling that $\gamma=\min \{a, b\}$, it follows that

$$
\begin{aligned}
\frac{\mathrm{d}}{\mathrm{d} t}\left(\|\widetilde{U}\|_{L^{4}}^{4}+G\|\widetilde{V}\|_{L^{4}}^{4}\right) & \\
& +(\gamma-4 \sigma z)\left(\|\widetilde{U}\|_{L^{4}}^{4}+G\|\widetilde{V}\|_{L^{4}}^{4}\right) \\
\leq & e^{-4 \sigma z\left(\theta_{s} \omega\right)} C_{2} \\
& +e^{-4 \sigma z\left(\theta_{s} \omega\right)}\left(\frac{8\left\|f_{1}\right\|_{L^{4}}^{4}}{a^{3}}+\frac{8\left\|f_{2}\right\|_{L^{4}}^{4}}{b^{3} G^{3}}\right),
\end{aligned}
$$

where $C_{2}=8 \rho^{4}|\Gamma| / a^{3}+8 \beta^{4}|\Gamma| / b^{3} G^{3}$. By Gronwall's lemma on the interval $\left[t_{0}, q\right], q \in[\tau-1, \tau]$, for the above inequality, and replacing $\omega$ by $\theta_{-\tau} \omega$, we get

$$
\begin{aligned}
& \left\|\widetilde{U}\left(q, \tau-t, \theta_{-\tau} \omega, \widetilde{U}_{\tau-t}\right)\right\|_{L^{4}}^{4} \\
& +G \| \widetilde{V}(q, \tau-t, \\
& \left.\theta_{-\tau} \omega, \widetilde{V}_{\tau-t}\right) \|_{L^{4}}^{4} \\
& \leq e^{\int_{t_{0}-\tau}^{q-\tau} 4 \sigma z\left(\theta_{s} \omega\right) \mathrm{d} s-\gamma\left(q-t_{0}\right)}\left(\| \widetilde{U}\left(t_{0}, \tau\right.\right. \\
& \left.-t, \theta_{-\tau} \omega, \widetilde{U}_{\tau-t}\right)\left\|_{L^{4}}^{4}+G\right\| \widetilde{V}\left(t_{0}, \tau-t, \theta_{-\tau} \omega,\right. \\
& \left.\left.\widetilde{V}_{\tau-t}\right) \|_{L^{4}}^{4}\right) \\
& +C_{2} \int_{-\infty}^{q-\tau} e^{-4 \sigma z\left(\theta_{s} \omega\right)+\int_{s}^{q-\tau} 4 \sigma z\left(\theta_{s} \omega\right) \mathrm{d} s+\gamma(s-q+\tau)} \mathrm{d} s \\
& +\frac{8}{a^{3}} \int_{-\infty}^{q-\tau} e^{-4 \sigma z\left(\theta_{s} \omega\right)+\int_{s}^{q-\tau} 4 \sigma z\left(\theta_{s} \omega\right) \mathrm{d} s+\gamma(s-q+\tau)} \| f_{1}(s \\
& +\tau) \|_{L^{4}}^{4} \mathrm{~d} s \\
& +\frac{8}{b^{3} G^{3}} \int_{-\infty}^{q-\tau} e^{-4 \sigma z\left(\theta_{s} \omega\right)+\int_{s}^{q-\tau} 4 \sigma z\left(\theta_{s} \omega\right) \mathrm{d} s+\gamma(s-q+\tau)} \| f_{2}(s \\
& +\tau) \|_{L^{4}}^{4} \mathrm{~d} s .
\end{aligned}
$$

Recalling that $\widetilde{U}=U, \widetilde{V}=V / G$, we have

$$
\begin{aligned}
& \left\|U\left(q, \tau-t, \theta_{-\tau} \omega, U_{\tau-t}\right)\right\|_{L^{4}}^{4} \\
& +G^{-3} \| V(q, \tau-t, \\
& \left.\theta_{-\tau} \omega, V_{\tau-t}\right) \|_{L^{4}}^{4} \leq 1 \\
& +C_{2} \int_{-\infty}^{q-\tau} e^{-4 \sigma z\left(\theta_{s} \omega\right)+\int_{s}^{q-\tau} 4 \sigma z\left(\theta_{s} \omega\right) \mathrm{d} s+\gamma(s-q+\tau)} \mathrm{d} s \\
& +\frac{8}{a^{3}} \int_{-\infty}^{q-\tau} e^{-4 \sigma z\left(\theta_{s} \omega\right)+\int_{s}^{q-\tau} 4 \sigma z\left(\theta_{s} \omega\right) \mathrm{d} s+\gamma(s-q+\tau)} \| f_{1}(s \\
& +\tau) \|_{L^{4}}^{4} \mathrm{~d} s
\end{aligned}
$$

$$
\begin{aligned}
& +\frac{8}{b^{3} G^{3}} \int_{-\infty}^{q-\tau} e^{-4 \sigma z\left(\theta_{s} \omega\right)+\int_{s}^{q-\tau} 4 \sigma z\left(\theta_{s} \omega\right) \mathrm{d} s+\gamma(s-q+\tau)} \| f_{2}(s \\
& +\tau) \|_{L^{4}}^{4} \mathrm{~d} s \doteq M_{3}(\tau, \omega) .
\end{aligned}
$$

Similarly, multiplying (65) by $\widetilde{U}^{5}$ and (66) by $G \widetilde{V}^{5}$, we get

$$
\begin{aligned}
\frac{\mathrm{d}}{\mathrm{d} t}\left(\|\widetilde{U}\|_{L^{6}}^{6}+G\|\widetilde{V}\|_{L^{6}}^{6}\right) & +\left(\gamma-6 \sigma z\left(\theta_{s} \omega\right)\right)\left(\|\widetilde{U}\|_{L^{6}}^{6}+G\|\widetilde{V}\|_{L^{6}}^{6}\right) \\
\leq & e^{-6 \sigma z\left(\theta_{s} \omega\right)}\left(\frac{32 \rho^{6}|\Gamma|}{a^{5}}+\frac{32 \beta^{6}|\Gamma|}{b^{5} G^{5}}\right) \\
& +e^{-6 \sigma z\left(\theta_{s} \omega\right)}\left(\frac{32\left\|f_{1}\right\|_{L^{6}}^{6}}{a^{5}}+\frac{32\left\|f_{2}\right\|_{L^{6}}^{6}}{b^{5} G^{5}}\right) .
\end{aligned}
$$

Put $C_{3}=32 \rho^{6}|\Gamma| / a^{5}+32 \beta^{6}|\Gamma| / b^{5} G^{5}$. Applying Gronwall's inequality over $\left[t_{0}, q\right]$ and replacing $\omega$ by $\theta_{-\tau} \omega$, we have

$$
\begin{aligned}
& \left\|\widetilde{U}\left(q, \tau-t, \theta_{-\tau} \omega, \widetilde{U}_{\tau-t}\right)\right\|_{L^{6}}^{6}+G \| \widetilde{V}\left(q, \tau-t, \theta_{-\tau} \omega\right. \\
& \left.\widetilde{V}_{\tau-t}\right) \|_{L^{6}}^{6} \leq e^{\int_{t_{0}}^{q} 6 \sigma z\left(\theta_{s-\tau} \omega\right) \mathrm{d} s-\gamma\left(q-t_{0}\right)}\left(\| \widetilde{U}\left(t_{0}, \tau\right.\right. \\
& \left.-t, \theta_{-\tau} \omega, \widetilde{U}_{\tau-t}\right)\left\|_{L^{6}}^{6}+G\right\| \widetilde{V}\left(t_{0}, \tau\right. \\
& \left.\left.-t, \theta_{-\tau} \omega, \widetilde{V}_{\tau-t}\right) \|_{L^{6}}^{6}\right) \\
& +C_{3} \int_{t_{0}}^{q} e^{-6 \sigma z\left(\theta_{s-\tau} \omega\right)+\int_{s}^{q} 6 \sigma z\left(\theta_{s-\tau} \omega\right) \mathrm{d} s-\gamma(q-s)} \mathrm{d} s+\frac{32}{a^{5}} \\
& \cdot \int_{t_{0}}^{q} e^{-6 \sigma z\left(\theta_{s-\tau} \omega\right)+\int_{s}^{q} 6 \sigma z\left(\theta_{s-\tau} \omega\right) \mathrm{d} s-\gamma(q-s)}\left\|f_{1}(s)\right\|_{L^{6}}^{6} \mathrm{~d} s \\
& +\frac{32}{b^{5} G^{5}} \\
& \quad \cdot \int_{t_{0}}^{q} e^{-6 \sigma z\left(\theta_{s-\tau} \omega\right)+\int_{s}^{q} 6 \sigma z\left(\theta_{s-\tau} \omega\right) \mathrm{d} s-\gamma(q-s)}\left\|f_{2}(s)\right\|_{L^{6}}^{6} \mathrm{~d} s .
\end{aligned}
$$

Recalling that $\widetilde{U}=U, \widetilde{V}=V / G$, we get

$$
\begin{aligned}
& \left\|U\left(q, \tau-t, \theta_{-\tau} \omega, U_{\tau-t}\right)\right\|_{L^{6}}^{6} \\
& +G^{-5} \| V(q, \tau-t \\
& \left.\theta_{-\tau} \omega, V_{\tau-t}\right) \|_{L^{6}}^{6} \leq 1 \\
& +C_{3} \int_{-\infty}^{q-\tau} e^{-6 \sigma z\left(\theta_{s} \omega\right)+\int_{s}^{q-\tau} 6 \sigma z\left(\theta_{s} \omega\right) \mathrm{d} s+\gamma(s-q+\tau)} \mathrm{d} s \\
& +\frac{32}{a^{5}} \int_{-\infty}^{q-\tau} e^{-6 \sigma z\left(\theta_{s} \omega\right)+\int_{s}^{q-\tau} 6 \sigma z\left(\theta_{s} \omega\right) \mathrm{d} s+\gamma(s-q+\tau)} \| f_{1}(s \\
& +\tau) \|_{L^{6}}^{6} \mathrm{~d} s
\end{aligned}
$$




$$
\begin{aligned}
& +\frac{32}{b^{5} G^{5}} \int_{-\infty}^{q-\tau} e^{-6 \sigma z\left(\theta_{s} \omega\right)+\int_{s}^{q-\tau} 6 \sigma z\left(\theta_{s} \omega\right) \mathrm{d} s+\gamma(s-q+\tau)} \| f_{2}(s \\
& +\tau) \|_{L^{6}}^{6} \mathrm{~d} s \doteq M_{4}(\tau, \omega) .
\end{aligned}
$$

The proof is completed.

Lemma 7. Suppose that $f_{i}(t, x) \in X, i=1,2$. Then, for every $\tau \in \mathbf{R}, \omega \in \Omega$, and $B=\{B(\tau, \omega): \tau \in \mathbf{R}, \omega \in \Omega\} \in \mathscr{D}$, there exist a number $T_{6}(\tau, \omega, B, \sigma) \geq 0$ and a tempered random variable $M_{5}(\tau, \omega)$

$$
\begin{aligned}
& M_{5}(\tau, \omega) \\
& =\left(1+2 \sigma \max _{-1 \leq s \leq 0}\left|z\left(\theta_{s} \omega\right)\right|-2 \gamma\right) M_{1} \\
& \quad+M_{4} \int_{-1}^{0} \frac{4 e^{4 \sigma z\left(\theta_{s} \omega\right)}}{3 d}\left(2+3 G^{2}\right) d s \\
& \quad+\int_{-1}^{0} e^{-2 \sigma z\left(\theta_{s} \omega\right)}|\Gamma| \frac{\rho^{2}+\beta^{2}}{d} d s \\
& \quad+\int_{-1}^{0} e^{-2 \sigma z\left(\theta_{s} \omega\right)} \frac{\left\|f_{1}(s+\tau)\right\|^{2}+\left\|f_{2}(s+\tau)\right\|^{2}}{d} d s .
\end{aligned}
$$

such that for all $t \geq T_{6}$, the solution $g$ of (19)-(22) with $g_{\tau-t} \in$ $B\left(\tau-t, \theta_{-t} \omega\right)$ satisfies

$$
\begin{aligned}
& \left\|\nabla U\left(\tau, \tau-t, \theta_{-\tau} \omega, U_{\tau-t}\left(\theta_{-\tau} \omega\right)\right)\right\|^{2} \\
& \quad+\left\|\nabla V\left(\tau, \tau-t, \theta_{-\tau} \omega, V_{\tau-t}(\omega)\right)\right\|^{2} \leq M_{5}(\tau, \omega),
\end{aligned}
$$

where $g_{\tau-t}=\left(U_{\tau-t}, V_{\tau-t}\right) \in B\left(\tau-t, \theta_{-t} \omega\right)$.

Proof. Multiplying (19) by $-\triangle U$ and (20) by $-\triangle V$, we have

$$
\begin{aligned}
\frac{1}{2} \frac{\mathrm{d}}{\mathrm{d} t}\|\nabla U\|^{2}= & -d_{1}\|\Delta U\|^{2}+\left(\rho e^{-\sigma z\left(\theta_{t} \omega\right)},-\Delta U\right) \\
& -a\|\nabla U\|^{2}-\left(e^{2 \sigma z\left(\theta_{t} \omega\right)} U^{2} V, \triangle U\right) \\
& +\left(G e^{2 \sigma z\left(\theta_{t} \omega\right)} U^{3}, \Delta U\right) \\
& +\left(e^{-\sigma z\left(\theta_{t} \omega\right)} f_{1},-\Delta U\right) \\
& +\sigma z\left(\theta_{t} \omega\right)\|\nabla U\|^{2}, \\
\frac{1}{2} \frac{\mathrm{d}}{\mathrm{d} t}\|\nabla V\|^{2}= & -d_{2}\|\Delta V\|^{2}+\left(\beta e^{-\sigma z\left(\theta_{t} \omega\right)},-\Delta V\right) \\
& -b\|\nabla V\|^{2}+\left(e^{2 \sigma z\left(\theta_{t} \omega\right)} U^{2} V, \triangle V\right) \\
& -\left(G e^{2 \sigma z\left(\theta_{t} \omega\right)} U^{3}, \triangle V\right) \\
& +\left(e^{-\sigma z\left(\theta_{t} \omega\right)} f_{2},-\Delta V\right) \\
& +\sigma z\left(\theta_{t} \omega\right)\|\nabla V\|^{2} .
\end{aligned}
$$

Then summing up the resulting equalities, we have

$$
\begin{aligned}
\frac{1}{2} \frac{\mathrm{d}}{\mathrm{d} t} & \left(\|\nabla U\|^{2}+\|\nabla V\|^{2}\right)+d_{1}\|\Delta U\|^{2}+d_{2}\|\Delta V\|^{2} \\
& +a\|\nabla U\|^{2}+b\|\nabla V\|^{2} \\
& -\sigma z\left(\theta_{t} \omega\right)\left(\|\nabla U\|^{2}+\|\nabla V\|^{2}\right) \\
= & e^{-\sigma z\left(\theta_{t} \omega\right)} \int_{\Gamma}(-\rho \triangle U-\beta \Delta V) \mathrm{d} x \\
& +e^{-\sigma z\left(\theta_{t} \omega\right)} \int_{\Gamma}\left(-f_{1} \Delta U-f_{2} \Delta V\right) \\
& +e^{2 \sigma z\left(\theta_{t} \omega\right)} \int_{\Gamma}\left(-U^{2} V \triangle U+G U^{3} \triangle U\right) \mathrm{d} x \\
& +e^{2 \sigma z\left(\theta_{t} \omega\right)} \int_{\Gamma}\left(U^{2} V \triangle V-G U^{3} \triangle V\right) \mathrm{d} x .
\end{aligned}
$$

Due to Hölder's inequality and $\epsilon$-Young inequality, we obtain

$$
\begin{gathered}
e^{-\sigma z\left(\theta_{t} \omega\right)} \int_{\Gamma}(-\rho \Delta U-\beta \Delta V) \mathrm{d} x \\
\leq e^{-2 \sigma z\left(\theta_{t} \omega\right)}\left(\frac{\rho^{2}|\Omega|}{d_{1}}+\frac{\beta^{2}|\Omega|}{d_{2}}\right)+\frac{d_{1}}{4}\|\Delta U\|^{2} \\
+\frac{d_{2}}{4}\|\Delta V\|^{2} . \\
e^{-\sigma z\left(\theta_{t} \omega\right)} \int_{\Gamma}\left(-f_{1} \Delta U-f_{2} \Delta V\right) \\
\leq e^{-2 \sigma z\left(\theta_{t} \omega\right)}\left(\frac{\left\|f_{1}\right\|^{2}}{d_{1}}+\frac{\left\|f_{2}\right\|^{2}}{d_{2}}\right)+\frac{d_{1}}{4}\|\Delta U\|^{2} \\
+\frac{d_{2}}{4}\|\Delta V\|^{2} .
\end{gathered}
$$

For the third term on the right-hand side of (82), we have

$$
\begin{aligned}
& e^{2 \sigma z\left(\theta_{t} \omega\right)} \int_{\Gamma}\left(-U^{2} V \triangle U+G U^{3} \triangle U\right) \mathrm{d} x \leq \frac{e^{4 \sigma z\left(\theta_{t} \omega\right)}}{d_{1}} \\
& \cdot \int_{\Gamma} U^{4} V^{2} \mathrm{~d} x+\frac{e^{4 \sigma z\left(\theta_{t} \omega\right)}}{d_{1}} \int_{\Gamma} G^{2} U^{6} \mathrm{~d} x+\frac{d_{1}}{4}\|\Delta U\|^{2} \\
& +\frac{d_{1}}{4}\|\Delta U\|^{2} \leq \frac{d_{1}}{2}\|\Delta U\|^{2} \\
& +\frac{e^{4 \sigma z\left(\theta_{t} \omega\right)}}{3 d_{1}}\left(\int_{\Gamma} 3 G^{2} U^{6} \mathrm{~d} x\right. \\
& \left.+\int_{\Gamma}\left(U^{6}+U^{6}+V^{6}\right) \mathrm{d} x\right) \leq \frac{d_{1}}{2}\|\triangle U\|^{2} \\
& +\frac{e^{4 \sigma z\left(\theta_{t} \omega\right)}}{3 d_{1}}\left(2+3 G^{2}\right)\left(\|U\|_{L^{6}}^{6}+\|V\|_{L^{6}}^{6}\right) .
\end{aligned}
$$


For the last term in (82), we get

$$
\begin{aligned}
& e^{2 \sigma z\left(\theta_{t} \omega\right)} \int_{\Gamma}\left(U^{2} V \triangle V-G U^{3} \Delta V\right) \mathrm{d} x \leq \frac{e^{4 \sigma z\left(\theta_{t} \omega\right)}}{d_{2}} \\
& \cdot \int_{\Gamma} U^{4} V^{2} \mathrm{~d} x+\frac{e^{4 \sigma z\left(\theta_{t} \omega\right)}}{d_{2}} \int_{\Gamma} G^{2} U^{6} \mathrm{~d} x+\frac{d_{2}}{4}\|\Delta V\|^{2} \\
& +\frac{d_{2}}{4}\|\Delta V\|^{2} \leq \frac{d_{2}}{2}\|\Delta V\|^{2} \\
& +\frac{e^{4 \sigma z\left(\theta_{t} \omega\right)}}{3 d_{2}}\left(\int_{\Gamma} 3 G^{2} U^{6} \mathrm{~d} x\right. \\
& \left.+\int_{\Gamma}\left(U^{6}+U^{6}+V^{6}\right) \mathrm{d} x\right) \leq \frac{d_{2}}{2}\|\Delta V\|^{2} \\
& +\frac{e^{4 \sigma z\left(\theta_{t} \omega\right)}}{3 d_{2}}\left(2+3 G^{2}\right)\left(\|U\|_{L^{6}}^{6}+\|V\|_{L^{6}}^{6}\right) .
\end{aligned}
$$

Recalling that $d=\min \left\{d_{1}, d_{2}\right\}$ and $\gamma=\min \{a, b\}$, it follows that

$$
\begin{aligned}
& \frac{\mathrm{d}}{\mathrm{d} t}\left(\|\nabla U\|^{2}+\|\nabla V\|^{2}\right)+\left(2 \gamma-2 \sigma z\left(\theta_{t} \omega\right)\right) \\
& \cdot\left(\|\nabla U\|^{2}+\|\nabla V\|^{2}\right) \leq \frac{2 e^{4 \sigma z\left(\theta_{t} \omega\right)}}{3}\left(\frac{1}{d_{1}}+\frac{1}{d_{2}}\right) \\
& \cdot\left(2+3 G^{2}\right)\left(\|U\|_{L^{6}}^{6}+\|V\|_{L^{6}}^{6}\right)+2 e^{-2 \sigma z\left(\theta_{t} \omega\right)}|\Gamma| \\
& \cdot\left(\frac{\rho^{2}}{d_{1}}+\frac{\beta^{2}}{d_{2}}\right)+2 e^{-2 \sigma z\left(\theta_{t} \omega\right)}\left(\frac{\left\|f_{1}\right\|^{2}}{d_{1}}+\frac{\left\|f_{2}\right\|^{2}}{d_{2}}\right) \\
& \leq \frac{4 e^{4 \sigma z\left(\theta_{t} \omega\right)}}{3 d}\left(2+3 G^{2}\right)\left(\|U\|_{L^{6}}^{6}+\|V\|_{L^{6}}^{6}\right) \\
& +2 e^{-2 \sigma z\left(\theta_{t} \omega\right)}|\Gamma| \frac{\rho^{2}+\beta^{2}}{d}+2 e^{-2 \sigma z\left(\theta_{t} \omega\right)} \\
& . \\
& \quad \frac{\left\|f_{1}\right\|^{2}+\left\|f_{2}\right\|^{2}}{d} .
\end{aligned}
$$

For $t \geq T_{5}(\tau, \omega, B) \geq 1$ and $s \in[\tau-1, \tau]$, we integrate (86) over $[s, \tau]$ to get

$$
\begin{aligned}
& \left\|\nabla U\left(\tau, \tau-t, \theta_{-\tau} \omega, U_{\tau-t}\right)\right\|^{2}+\| \nabla V(\tau, \tau \\
& \left.-t, \theta_{-\tau} \omega, V_{\tau-t}\right)\left\|^{2} \leq\right\| \nabla U\left(s, \tau-t, \theta_{-\tau} \omega, U_{\tau-t}\right) \|^{2} \\
& +\left\|\nabla V\left(s, \tau-t, \theta_{-\tau} \omega, V_{\tau-t}\right)\right\|^{2}+\int_{\tau-1}^{\tau} 2\left(\sigma z\left(\theta_{s-\tau} \omega\right)\right. \\
& -\gamma)\left(\left\|\nabla U\left(s, \tau-t, \theta_{-\tau} \omega, U_{\tau-t}\right)\right\|^{2}\right. \\
& \left.+\left\|\nabla V\left(s, \tau-t, \theta_{-\tau} \omega, V_{\tau-t}\right)\right\|^{2}\right) \mathrm{d} s \\
& +\int_{\tau-1}^{\tau} \frac{4 e^{4 \sigma z\left(\theta_{s-\tau} \omega\right)}}{3 d}\left(2+3 G^{2}\right)\left(\|U\|_{L^{6}}^{6}+\|V\|_{L^{6}}^{6}\right) \mathrm{d} s
\end{aligned}
$$

$$
\begin{aligned}
& +\int_{\tau-1}^{\tau} 2 e^{-2 \sigma z\left(\theta_{s-\tau} \omega\right)}|\Gamma| \frac{\rho^{2}+\beta^{2}}{d} \mathrm{~d} s \\
& +\int_{\tau-1}^{\tau} 2 e^{-2 \sigma z\left(\theta_{s-\tau} \omega\right)} \frac{\left\|f_{1}\right\|^{2}+\left\|f_{2}\right\|^{2}}{d} \mathrm{~d} s .
\end{aligned}
$$

Now integrating the above with respect to $s$ over $(\tau-1, \tau)$, we find that for $t \geq T_{6}(\tau, \omega, B, \sigma)$, it follows that

$$
\begin{aligned}
& \left\|\nabla U\left(\tau, \tau-t, \theta_{-\tau} \omega, U_{\tau-t}\right)\right\|^{2}+\| \nabla V(\tau, \tau \\
& \left.-t, \theta_{-\tau} \omega, V_{\tau-t}\right) \|^{2} \leq\left(1+2 \sigma \max _{-1 \leq s \leq 0}\left|z\left(\theta_{s} \omega\right)\right|-2 \gamma\right) \\
& \cdot \int_{\tau-1}^{\tau}\left(\left\|\nabla U\left(s, \tau-t, \theta_{-\tau} \omega, U_{\tau-t}\right)\right\|^{2}\right. \\
& \left.+\left\|\nabla V\left(s, \tau-t, \theta_{-\tau} \omega, V_{\tau-t}\right)\right\|^{2}\right) \mathrm{d} s \\
& +\int_{\tau-1}^{\tau} \frac{4 e^{4 \sigma z\left(\theta_{s-\tau} \omega\right)}}{3 d}\left(2+3 G^{2}\right)\left(\|U(s)\|_{L^{6}}^{6}\right. \\
& \left.+\|V(s)\|_{L^{6}}^{6}\right) \mathrm{d} s+\int_{\tau-1}^{\tau} 2 e^{-2 \sigma z\left(\theta_{s-\tau} \omega\right)}|\Gamma| \\
& +\frac{\rho^{2}+\beta^{2}}{d} \mathrm{~d} s+\int_{\tau-1}^{\tau} 2 e^{-2 \sigma z\left(\theta_{s-\tau} \omega\right)} \\
& +\frac{\left\|f_{1}(s)\right\|^{2}+\left\|f_{2}(s)\right\|^{2}}{d} \mathrm{~d} s .
\end{aligned}
$$

By the Mean Value Theorem in (60), there is a time $t_{0} \in[\tau-$ $2, \tau-1]$ such that

$$
\begin{aligned}
& \left\|\nabla U\left(t_{0}, \tau-t, \theta_{-\tau} \omega, U_{\tau-t}\right)\right\|^{2} \\
& \quad+\left\|\nabla V\left(t_{0}, \tau-t, \theta_{-\tau} \omega, V_{\tau-t}\right)\right\|^{2} \leq M_{2}(\tau, \omega) .
\end{aligned}
$$

Then by Lemma 6 , for $q \in[\tau-1, \tau]$, there is a random variable $M_{4}(\tau, \omega)>0$ such that

$$
\begin{aligned}
& \left\|U\left(q, \tau-t, \theta_{-\tau} \omega, U_{\tau-t}\right)\right\|_{L^{6}}^{6} \\
& \quad+\left\|V\left(q, \tau-t, \theta_{-\tau} \omega, U_{\tau-t}\right)\right\|_{L^{6}}^{6} \leq M_{4}(\tau, \omega) .
\end{aligned}
$$

By Lemma 5, it follows from (77) that, for all $t \geq T_{5}(\tau, \omega, B, \sigma)$,

$$
\begin{aligned}
& \left\|\nabla U\left(\tau, \tau-t, \theta_{-\tau} \omega, U_{\tau-t}\right)\right\|^{2} \\
& \quad+\left\|\nabla V\left(\tau, \tau-t, \theta_{-\tau} \omega, V_{\tau-t}\right)\right\|^{2}
\end{aligned}
$$




$$
\begin{aligned}
& \leq\left(1+2 \sigma \max _{-1 \leq s \leq 0}\left|z\left(\theta_{s} \omega\right)\right|-2 \gamma\right) M_{1} \\
& +M_{4} \int_{-1}^{0} \frac{4 e^{4 \sigma z\left(\theta_{s} \omega\right)}}{3 d}\left(2+3 G^{2}\right) \mathrm{d} s \\
& +\int_{-1}^{0} e^{-2 \sigma z\left(\theta_{s} \omega\right)}|\Gamma| \frac{\rho^{2}+\beta^{2}}{d} \mathrm{~d} s \\
& +\int_{-1}^{0} e^{-2 \sigma z\left(\theta_{s} \omega\right)} \frac{\left\|f_{1}(s+\tau)\right\|^{2}+\left\|f_{2}(s+\tau)\right\|^{2}}{d} \mathrm{~d} s \\
& \doteq M_{5}(\tau, \omega) .
\end{aligned}
$$

It is easy to see that $M_{5}(\tau, \omega)$ is tempered. This completes the proof.

Lemma 8. Suppose that $f_{i}(t, x) \in X(i=1,2)$. Then for each $\tau \in \mathbf{R}$ and $\omega \in \Omega$, the continuous cocycle $\varphi$ associated with problem (19)-(22) has a closed measurable D-pullback absorbing set $B_{\sigma}(\tau, \omega) \in \mathscr{D}$, which is given by

$$
B_{\sigma}(\tau, \omega)=\left\{(U, V) \in H:\|(U, V)\|^{2} \leq R_{\sigma}(\tau, \omega)\right\},
$$

where $R_{\sigma}(\tau, \omega)$ is defined as in (32).

Theorem 9. Suppose that $f_{i}(t, x) \in X(i=1,2)$. Then the continuous cocycle $\varphi$ associated with problem (19)-(22) has a unique $\mathscr{D}$-pullback attractor $\mathscr{A}_{\sigma}=\left\{\mathscr{A}_{\sigma}(\tau, \omega): \tau \in \mathbf{R}, \omega \in \Omega\right\}$ in $\mathrm{H}$.

Proof. By Lemma 8, the continuous cocycle $\varphi$ has a $\mathscr{D}$ pullback absorbing set $B_{\tau}(\sigma, \omega)$. By the compact embedding $E \hookrightarrow H$, the continuous cocycle $\varphi$ is pullback asymptotically compact in $H$. According to Theorem 1 , there exists a unique random attractor $\mathscr{A}_{\sigma}$ in $H$ for the continuous cocycle $\varphi$.

\section{Upper Semicontinuity of Random Attractors}

In this section, we prove the upper semicontinuity of random pullback attractors for the nonautonomous stochastic reversible Selkov system when the intensity of noise $\sigma \longrightarrow 0$. Given $\sigma \in[0,1]$, we write the cocycle of problem (19)-(22) as $\varphi_{\sigma}$ to indicate its dependence on $\sigma$.

The deterministic system corresponding to (19)-(22) is given by

$$
\begin{aligned}
& \frac{d u}{d t}=d_{1} \Delta u+\rho-a u+u^{2} v-G u^{3}+f_{1}(t, x), \\
& \frac{d v}{d t}=d_{2} \Delta v+\beta-b v-u^{2} v+G u^{3}+f_{2}(t, x),
\end{aligned}
$$

with the homogeneous Dirichlet boundary conditions

$$
u(t, x)=v(t, x)=0, \quad t>\tau, x \in \partial \Gamma,
$$

and the initial conditions

$$
\begin{gathered}
u(\tau, x)=u_{\tau}(x), \\
v(\tau, x)=v_{\tau}(x),
\end{gathered}
$$

Similarly, we can prove that problem (93)-(96) generates a continuous cocycle $\varphi_{0}$ in $H$. Moreover, $\varphi_{0}$ has a unique pullback attractor $\mathscr{A}_{0}=\left\{\mathscr{A}_{0}(\tau), \tau \in \mathbf{R}\right\}$ in $H$ and has a pullback absorbing set $B_{0}(\tau)=\left\{B_{0}(\tau), \tau \in \mathbf{R}\right\}$, where $B_{0}(\tau)$ is given by

$$
B_{0}(\tau)=\left\{(u, v) \in H:\|(u, v)\|^{2} \leq R_{0}(\tau)\right\}
$$

and

$$
\begin{aligned}
R_{0}(\tau)= & 1+C_{1} \int_{-\infty}^{0} e^{\gamma s} \mathrm{~d} s \\
& +\frac{G}{a} \int_{-\infty}^{0} e^{\gamma s}\left\|f_{1}(s+\tau)\right\|^{2} \mathrm{~d} s \\
& +\frac{1}{b} \int_{-\infty}^{0} e^{\gamma s}\left\|f_{2}(s+\tau)\right\|^{2} \mathrm{~d} s .
\end{aligned}
$$

The next lemma shows the convergence of solutions of (19)-(22) as $\sigma \longrightarrow 0$.

Lemma 10. Suppose that $f_{i}(t, x) \in X(i=1,2)$. Let $g_{\sigma}=$ $(U, V)$ and $g=(u, v)$ be the solution of (19)-(22) and (93)-(96) with initial conditions $g_{\tau, \sigma}$ and $g_{\tau}$, respectively. If $\lim _{\sigma \longrightarrow 0} g_{\sigma, \tau}=g_{\tau}$, then, for $\omega \in \Omega, t>\tau$ and $\varepsilon \in[0,1]$,

$$
\lim _{\sigma \longrightarrow 0} g_{\sigma}\left(t, \tau, \omega, g_{\tau, \sigma}\right)=g\left(t, \tau, g_{\tau}\right)
$$

Proof. Let $U$ and $V$ be the solution of (19)-(22), and $\tilde{u}=U-u$, $\widetilde{v}=V-v$, and $k=(\tilde{u}, \widetilde{v})=(U-u, V-v)=g_{\sigma}-g$. Then from (19)-(20) and (93)-(94), we get

$$
\begin{aligned}
\frac{d \tilde{u}}{d t}= & d_{1} \Delta \tilde{u}+\left(e^{-\sigma z\left(\theta_{t} \omega\right)}-1\right) \rho-a \tilde{u}+e^{2 \sigma z\left(\theta_{t} \omega\right)} U^{2} V \\
& -u^{2} v-G e^{2 \sigma z\left(\theta_{t} \omega\right)} U^{3}+G u^{3} \\
& +e^{-\sigma z\left(\theta_{t} \omega\right)} f_{1}(t, x)-f_{1}(t, x)+\sigma U z\left(\theta_{t} \omega\right), \\
\frac{d \widetilde{v}}{d t}= & d_{2} \Delta \widetilde{v}+\left(e^{-\sigma z\left(\theta_{t} \omega\right)}-1\right) \beta-b \widetilde{v}-e^{2 \sigma z\left(\theta_{t} \omega\right)} U^{2} V \\
& +u^{2} v+G e^{2 \sigma z\left(\theta_{t} \omega\right)} U^{3}-G u^{3} \\
& +e^{-\sigma z\left(\theta_{t} \omega\right)} f_{2}(t, x)-f_{2}(t, x)+\sigma V z\left(\theta_{t} \omega\right),
\end{aligned}
$$

which implies

$$
\begin{aligned}
& \frac{1}{2} \frac{\mathrm{d}}{\mathrm{d} t}\|\tilde{u}\|^{2}+d_{1}\|\nabla \tilde{u}\|^{2}+a\|\tilde{u}\|^{2}=\int_{\Gamma}\left(e^{-\sigma z\left(\theta_{t} \omega\right)}-1\right) \\
& \cdot \rho \tilde{u} \mathrm{~d} x+\int_{\Gamma}\left(e^{-\sigma z\left(\theta_{t} \omega\right)}-1\right) f_{1} \tilde{u} \mathrm{~d} x \\
& \quad+\int_{\Gamma}\left(e^{2 \sigma z\left(\theta_{t} \omega\right)} U^{2} V-u^{2} v-G e^{2 \sigma z\left(\theta_{t} \omega\right)} U^{3}+G u^{3}\right) \\
& \cdot \tilde{u} \mathrm{~d} x+\int_{\Gamma} \sigma z\left(\theta_{t} \omega\right) U \tilde{u} \mathrm{~d} x,
\end{aligned}
$$




$$
\begin{aligned}
& \frac{1}{2} \frac{\mathrm{d}}{\mathrm{d} t}\|\widetilde{v}\|^{2}+d_{2}\|\nabla \widetilde{v}\|^{2}+b\|\widetilde{v}\|^{2}=\int_{\Gamma}\left(e^{-\sigma z\left(\theta_{t} \omega\right)}-1\right) \\
& \cdot \beta \widetilde{v} \mathrm{~d} x+\int_{\Gamma}\left(e^{-\sigma z\left(\theta_{t} \omega\right)}-1\right) f_{2} \widetilde{v} \mathrm{~d} x \\
& \quad-\int_{\Gamma}\left(e^{2 \sigma z\left(\theta_{t} \omega\right)} U^{2} V-u^{2} v-G e^{2 \sigma z\left(\theta_{t} \omega\right)} U^{3}+G u^{3}\right) \\
& \cdot \widetilde{v} \mathrm{~d} x+\int_{\Gamma} \sigma z\left(\theta_{t} \omega\right) V \widetilde{v} \mathrm{~d} x .
\end{aligned}
$$

Summing up the two equalities together, it yields that

$$
\begin{aligned}
& \frac{\mathrm{d}}{\mathrm{d} t}\left(\|\widetilde{u}\|^{2}+\|\widetilde{v}\|^{2}\right)+2 d\left(\|\nabla \widetilde{u}\|^{2}+\|\nabla \widetilde{v}\|^{2}\right)+2 a\|\widetilde{u}\|^{2} \\
& \quad+2 b\|\widetilde{v}\|^{2}=2 \int_{\Gamma}\left(e^{-\sigma z\left(\theta_{t} \omega\right)}-1\right)(\rho \widetilde{u}+\beta \widetilde{v}) \mathrm{d} x \\
& \quad+2 \int_{\Gamma}\left(e^{-\sigma z\left(\theta_{t} \omega\right)}-1\right)\left(f_{1} \widetilde{u}+f_{2} \widetilde{v}\right) \mathrm{d} x \\
& \quad+2 \int_{\Gamma} \sigma z\left(\theta_{t} \omega\right)(U \widetilde{u}+V \widetilde{v}) \mathrm{d} x \\
& \quad+2 \int_{\Gamma}\left(e^{2 \sigma z\left(\theta_{t} \omega\right)} U^{2} V-u^{2} v-G e^{2 \sigma z\left(\theta_{t} \omega\right)} U^{3}+G u^{3}\right) \\
& \quad \cdot(\widetilde{u}-\widetilde{v}) \mathrm{d} x .
\end{aligned}
$$

Note that

$$
\begin{aligned}
& \int_{\Gamma}\left(e^{2 \sigma z\left(\theta_{t} \omega\right)} U^{2} V-u^{2} v-G e^{2 \sigma z\left(\theta_{t} \omega\right)} U^{3}+G u^{3}\right) \\
& \cdot(\widetilde{u}-\widetilde{v}) \mathrm{d} x=\int_{\Gamma} e^{2 \sigma z\left(\theta_{t} \omega\right)}\left(U^{2} V-G U^{3}\right) \\
& \cdot(\widetilde{u}-\widetilde{v}) \mathrm{d} x+\int_{\Gamma}\left(G u^{3}-u^{2} v\right)(\widetilde{u}-\widetilde{v}) \mathrm{d} x \\
& =\int_{\Gamma}\left(e^{2 \sigma z\left(\theta_{t} \omega\right)}-1\right)\left(U^{2} V-G U^{3}\right)(\widetilde{u}-\widetilde{v}) \mathrm{d} x \\
& +\int_{\Gamma}\left(U^{2} V-G U^{3}+G u^{3}-u^{2} v\right)(\widetilde{u}-\widetilde{v}) \mathrm{d} x \\
& \quad=\int_{\Gamma}\left(e^{2 \sigma z\left(\theta_{t} \omega\right)}-1\right)\left(U^{2} V-G U^{3}\right)(\widetilde{u}-\widetilde{v}) \mathrm{d} x \\
& \quad+\int_{\Gamma}\left[\left(U^{2} V-u^{2} v\right)-\left(G U^{3}-G u^{3}\right)\right] \\
& \cdot(\widetilde{u}-\widetilde{v}) \mathrm{d} x .
\end{aligned}
$$

Given that $\tau \in \mathbf{R}, \omega \in \Omega, T>0$, and $\varepsilon \in[0,1)$, since $\omega$ is continuous on $\mathbf{R}$, we obtain that there exists a $\sigma_{1}=$ $\sigma_{1}(\omega, T, \varepsilon)>0$ such that for all $\sigma \in\left(0, \sigma_{1}\right)$ and $t \in[\tau, \tau+T]$,

$$
\begin{gathered}
\left|e^{-\sigma z\left(\theta_{t} \omega\right)}-1\right|+\left|e^{\sigma z\left(\theta_{t} \omega\right)}-1\right|+\left|e^{2 \sigma z\left(\theta_{t} \omega\right)}-1\right| \\
+\left|e^{-2 \sigma z\left(\theta_{t} \omega\right)}-1\right|<\varepsilon .
\end{gathered}
$$

Thus, we obtain

$$
\begin{aligned}
& \frac{\mathrm{d}}{\mathrm{d} t}\left(\|\widetilde{u}\|^{2}+\|\widetilde{v}\|^{2}\right)+2 d\left(\|\nabla \widetilde{u}\|^{2}+\|\nabla \widetilde{v}\|^{2}\right)+2 a\|\widetilde{u}\|^{2} \\
& +2 b\|\widetilde{v}\|^{2} \leq a\|\widetilde{u}\|^{2}+b\|\widetilde{v}\|^{2}+\frac{\varepsilon^{2} \rho^{2}|\Gamma|}{a}+\frac{\varepsilon^{2} \beta^{2}|\Gamma|}{b} \\
& \quad+a\|\widetilde{u}\|^{2}+b\|\widetilde{v}\|^{2}+\frac{\varepsilon^{2}\left\|f_{1}\right\|^{2}}{a}+\frac{\varepsilon^{2}\left\|f_{2}\right\|^{2}}{b} \\
& \quad+2 \sigma z\left(\theta_{t} \omega\right) \int(|U \widetilde{u}|+|V \widetilde{v}|) \mathrm{d} x \\
& \quad+2 \varepsilon \int_{\Gamma}\left(\left|U^{2} V\right|+\left|G U^{3}\right|\right)(|\widetilde{u}|+|\widetilde{v}|) \mathrm{d} x \\
& \quad+2 \int_{\Gamma}\left(\left|U^{2} V-u^{2} v\right|+\left|G U^{3}-G u^{3}\right|\right) \\
& \quad+(|\widetilde{u}|+|\widetilde{v}|) \mathrm{d} x .
\end{aligned}
$$

Lemma 6 is true even if $q \in[\tau, t]$. By Hölder's inequality and Gagliardo-Nirenberg inequality, we have

$$
\begin{gathered}
\int_{\Gamma}\left(\left|U^{2} V-u^{2} v\right|+\left|G U^{3}-G u^{3}\right|\right)(|\widetilde{u}|+|\widetilde{v}|) \mathrm{d} x \\
\quad \leq d(\|\nabla \widetilde{u}\|+\|\nabla \widetilde{v}\|)+c_{1}^{\prime}\left(\|g\|+\|g\|_{L^{4}}^{2}+\|g\|_{L^{6}}^{3}\right. \\
\left.\quad+\left\|g_{\sigma}\right\|+\left\|g_{\sigma}\right\|_{L^{4}}^{2}+\left\|g_{\sigma}\right\|_{L^{6}}^{3}\right)\left(\|\widetilde{u}\|^{2}+\|\widetilde{v}\|^{2}\right),
\end{gathered}
$$

where $c_{1}^{\prime}$ is a constant depending on $d, G$ and the GagliardoNirenberg inequality constant. It follows from (105) and (106) that there exists a $\sigma_{2}=\sigma_{2}(\omega, T, \varepsilon)>0$ such that for all $\sigma \in$ $\left(0, \sigma_{2}\right)$ and $t \in[\tau, \tau+T]$,

$$
\begin{aligned}
& \frac{\mathrm{d}}{\mathrm{d} t}\left(\|\widetilde{u}\|^{2}+\|\widetilde{v}\|^{2}\right) \leq \frac{\varepsilon^{2} \rho^{2}|\Gamma|}{a}+\frac{\varepsilon^{2} \beta^{2}|\Gamma|}{b}+\frac{\varepsilon^{2}\left\|f_{1}\right\|^{2}}{a} \\
& +\frac{\varepsilon^{2}\left\|f_{2}\right\|^{2}}{b}+2 c_{2}^{\prime}\left(\|g\|+\|g\|_{L^{4}}^{2}+\|g\|_{L^{6}}^{3}+\left\|g_{\sigma}\right\|\right. \\
& \left.+\left\|g_{\sigma}\right\|_{L^{4}}^{2}+\left\|g_{\sigma}\right\|_{L^{6}}^{3}\right)\left(\|\widetilde{u}\|^{2}+\|\widetilde{v}\|^{2}\right) \leq c_{4} \varepsilon^{2} \\
& +\frac{\varepsilon^{2}\left\|f_{1}\right\|^{2}}{a}+\frac{\varepsilon^{2}\left\|f_{2}\right\|^{2}}{b}+2 c_{3}^{\prime},
\end{aligned}
$$

where $c_{4}=2 \rho^{2}|\Gamma| / a+2 \beta^{2}|\Gamma| / b, c_{3}^{\prime}=2 c_{2}^{\prime}\left(\|g\|+\|g\|_{L^{4}}^{2}+\|g\|_{L^{6}}^{3}+\right.$ $\left.\left\|g_{\sigma}\right\|+\left\|g_{\sigma}\right\|_{L^{4}}^{2}+\left\|g_{\sigma}\right\|_{L^{6}}^{3}\right)$. For all $\sigma \in\left(0, \sigma_{2}\right)$ and $t \in[\tau, \tau+T]$, due to Gronwall's lemma, we get

$$
\begin{aligned}
& \|k(t)\|^{2} \\
& \leq e^{\left.c_{3}^{\prime}(t-\tau)\right)}\left(\|\widetilde{u}(\tau)\|^{2}+\|\widetilde{v}(\tau)\|^{2}\right) \\
& \quad+\varepsilon^{2} \int_{\tau}^{t} e^{-c_{3}^{\prime}(t-s)}\left(c_{4}+\frac{\left\|f_{1}(r)\right\|^{2}}{a}+\frac{\left\|f_{2}(r)\right\|^{2}}{b}\right) \mathrm{d} r .
\end{aligned}
$$


From (108), recalling that $k=(\tilde{u}, \widetilde{v})=(U-u, V-v)=g_{\sigma}-g$, we find that

$$
\begin{aligned}
& \left\|g_{\sigma}\left(t, \tau, \omega, g_{\tau, \sigma}\right)-g\left(t, \tau, g_{\tau}\right)\right\|^{2} \\
& \leq e^{c_{3}^{\prime}(t-\tau)}\left(\left\|g_{\tau, \sigma}-g_{\tau}\right\|^{2}\right) \\
& \quad+\varepsilon^{2} \int_{\tau}^{t} e^{-c_{3}^{\prime}(t-s)}\left(c_{4}+\frac{\left\|f_{1}(r)\right\|^{2}}{a}+\frac{\left\|f_{2}(r)\right\|^{2}}{b}\right) \mathrm{d} r,
\end{aligned}
$$

which finishes the proof.

Theorem 11. Provided that $f_{i}(t, x) \in X(i=1,2)$. Then for every $\tau \in \mathbf{R}$ and $\omega \in \Omega$,

$$
\lim _{\sigma \longrightarrow 0} d_{H}\left(\mathscr{A}_{\sigma}(\tau, \omega), \mathscr{A}_{0}(\tau)\right)=0
$$

Proof. To achieve the result, it suffices to verify conditions (1), (2), and (3) in Proposition 3. For condition (1), by taking a sequence $\sigma_{n} \longrightarrow 0$ and $g_{\tau, n} \longrightarrow g_{\tau}$, from Lemma 10 we have

$$
\varphi_{\sigma_{n}}\left(t, \tau, \omega, g_{\tau, n}\right) \longrightarrow \varphi\left(t, \tau, g_{\tau}\right) \text { in } H \text {. }
$$

By Lemma 8 and Theorem $9, \varphi_{\sigma}$ has a tempered pullback absorbing set $B_{\sigma}$ and a unique $\mathscr{D}$-pullback random attractor $\mathscr{A}_{\sigma} \in \mathscr{D}$. Here,

$$
B_{\sigma}(\tau, \omega)=\left\{U, V \in H:\|(U, V)\|^{2} \leq R_{\sigma}(\tau, \omega)\right\},
$$

where $R_{\sigma}(\tau, \omega)$ is defined as in (32). It follows from (97) and (112) that for all $\tau \in \mathbf{R}$ and $\omega \in \Omega$, we have

$$
\limsup _{\sigma \rightarrow 0}\left\|B_{\sigma}(\tau, \omega)\right\| \leq\left\|B_{0}(\tau)\right\|,
$$

which deduces condition (2) immediately.

Now consider condition (3). By Lemma 7, we know that $\left\{K_{\sigma}(\tau, \omega)\right\} \in \mathscr{D}$ is also a closed and tempered absorbing set for $\varphi_{\sigma}$ in $H$, where

$$
K_{\sigma}(\tau, \omega)=\left\{(U, V) \in H:\|(U, V)\|_{E}^{2} \leq M_{5}(\tau, \omega)\right\} .
$$

Let

$$
\begin{aligned}
& \widetilde{M}_{5}(\tau, \omega) \\
& =\left(1+2 \max _{-1 \leq s \leq 0}\left|z\left(\theta_{s} \omega\right)\right|-2 \gamma\right) M_{1} \\
& \quad+M_{4} \int_{-1}^{0} \frac{4 e^{4\left|z\left(\theta_{s} \omega\right)\right|}}{3 d}\left(2+3 G^{2}\right) \mathrm{d} s \\
& \quad+\int_{-1}^{0} e^{2\left|z\left(\theta_{s} \omega\right)\right|}|\Gamma| \frac{\rho^{2}+\beta^{2}}{d} \mathrm{~d} s \\
& \quad+\int_{-1}^{0} e^{2\left|z\left(\theta_{s} \omega\right)\right|} \frac{\left\|f_{1}(s+\tau)\right\|^{2}+\left\|f_{2}(s+\tau)\right\|^{2}}{d} \mathrm{~d} s,
\end{aligned}
$$

and

$$
K(\tau, \omega)=\left\{(U, V) \in H:\|(U, V)\|_{E}^{2} \leq \widetilde{M}_{5}(\tau, \omega)\right\} .
$$

We find that for all $\tau \in \mathbf{R}$ and $\omega \in \Omega$,

$$
\bigcup_{0<\sigma \leq 1} \mathscr{A}_{\sigma}(\tau, \omega) \subseteq \bigcup_{0<\sigma \leq 1} K_{\sigma}(\tau, \omega) \subseteq K(\tau, \omega) .
$$

By the compactness of embedding $H \hookrightarrow E$, we know that $K(\tau, \omega)$ is compact in $H$. By (117), it follows that $\bigcup_{0<\sigma \leq 1} \mathscr{A}_{\sigma}(\tau, \omega)$ is precompact in $H$. Hence, condition (3) is clear and the proof is finished.

\section{Data Availability}

The data used to support the findings of this study are included within the article.

\section{Conflicts of Interest}

The authors declare that they have no conflicts of interest regarding the publication of this paper.

\section{Acknowledgments}

The authors would like to thank the reviewer for their valuable comments and suggestions which improve the manuscript. This paper was partially supported by the National Natural Science Foundation of China Nos. 11771444, 11861013, the Yue Qi Young Scholar Project, China University of Mining and Technology (Beijing), China Scholarship Council (CSC), Guangxi Natural Science Foundation No. 2017GXNSFAA198221, the Fundamental Research Funds for the Central Universities, and China University of Geosciences (Wuhan) No. 2018061.

\section{References}

[1] Y. You, "Asymptotical dynamics of Selkov equations," Discrete and Continuous Dynamical Systems - Series S, vol. 2, no. 1, pp. 193-219, 2009.

[2] Ed. L, Mathematical Models in Molecular and Cellular Biology, L. A. Segel, Ed., Cambridge University Press, 1980.

[3] E. E. Selkov, "Self-oscillations in glycolysis:a simple kinetic model," European Journal of Biochemistry, vol. 4, pp. 79-86, 1968.

[4] Y. You, "Global dynamics and robustness of reversible autocatalytic reaction-diffusion systems," Nonlinear Analysis. Theory, Methods \& Applications, vol. 75, no. 6, pp. 3049-3071, 2012.

[5] H. Li, "Random attractor of the stochastic lattice reversible selkov equations with additive noises," in Proceedings of the IEEE 13th International Conference on e-Business Engineering (ICEBE), pp. 176-181, 2016.

[6] L. Arnold, Random Dynamical Systems, Springer-Verlag, Berlin, Germany, 1998.

[7] P. W. Bates, H. Lisei, and K. Lu, "Attractors for stochastic lattice dynamical systems," Stochastics and Dynamics, vol. 6, no. 1, pp. 626-637, 2006.

[8] P. W. Bates, K. Lu, and B. Wang, "Random attractors for stochastic reaction-diffusion equations on unbounded domains," Journal of Differential Equations, vol. 246, no. 2, pp. 845-869, 2009.

[9] H. Crauel and F. Flandoli, "Attractors for random dynamical systems," Probability Theory and Related Fields, vol. 100, no. 3, pp. 365-393, 1994. 
[10] T. Caraballo, M. J. Garrido-Atienza, and T. Taniguchi, "The existence and exponential behavior of solutions to stochastic delay evolution equations with a fractional Brownian motion," Nonlinear Analysis. Theory, Methods \& Applications, vol. 74, no. 11, pp. 3671-3684, 2011.

[11] T. Caraballo and J. A. Langa, "On the upper semicontinuity of cocycle attractors for non-autonomous and random dynamical systems," Dynamics of Continuous, Discrete and Impulsive Systems Series A: Mathematical Analysis, vol. 10, no. 4, pp. 491-513, 2003.

[12] H. Cui and Y. Li, "Existence and upper semicontinuity of random attractors for stochastic degenerate parabolic equations with multiplicative noises," Applied Mathematics and Computation, vol. 271, pp. 777-789, 2015.

[13] I. Chueshov, Monotone Random Systems Theory and Applications, vol. 1779 of Lecture Notes in Mathematics, Springer, Berlin, Germany, 2002.

[14] I. Chueshov, J. Real, and T. Caraballo, "Pullback attractors for stochastic heat equations in materials with memory," Discrete and Continuous Dynamical Systems - Series B, vol. 9, no. 3/4, May, pp. 525-539, 2008.

[15] I. Chueshov and M. Scheutzow, "On the structure of attractors and invariant measures for a class of monotone random systems," Dynamical Systems, vol. 19, no. 2, pp. 127-144, 2004.

[16] F. Flandoli and B. Schmalfuss, "Random attractors for the 3D stochastic Navier-Stokes equation with multiplicative white noise," Stochastics and Stochastics Reports, vol. 59, no. 1-2, pp. 21-45, 1996.

[17] M. J. Garrido-Atienza, A. Ogrowsky, and B. Schmalfuss, "Random differential equations with random delays," Stochastics and Dynamics, vol. 11, pp. 369-388, 2011.

[18] M. J. Garrido-Atienza and B. Schmalfuss, "Ergodicity of the infinite dimensional fractional Brownian motion," Journal of Dynamics and Differential Equations, vol. 23, no. 3, pp. 671-681, 2011.

[19] W. Shen and J. Huang, "Pullback attractors for nonautonomous and random parabolic equations on non-smooth domains," Discrete and Continuous Dynamical Systems - Series A, vol. 24, no. 3, pp. 855-882, 2009.

[20] P. E. Kloeden and J. A. Langa, "Flattening, squeezing and the existence of random attractors," Proceedings of the Royal Society A Mathematical, Physical and Engineering Sciences, vol. 463, no. 2077, pp. 163-181, 2007.

[21] Y. Li, H. Cui, and J. Li, "Upper semi-continuity and regularity of random attractors on p-times integrable spaces and applications," Journal of Nonlinear Analysis, vol. 109, pp. 33-44, 2014.

[22] Y. Lv and W. Wang, "Limiting dynamics for stochastic wave equations," Journal of Differential Equations, vol. 244, no. 1, pp. 1-23, 2008.

[23] A. Gu, S. Zhou, and Z. Wang, "Uniform attractor of nonautonomous three-component reversible Gray-Scott system," Applied Mathematics and Computation, vol. 219, no. 16, pp. 8718-8729, 2013.

[24] A. Gu and H. Xiang, "Upper semicontinuity of random attractors for stochastic three-component reversible Gray-Scott system," Applied Mathematics and Computation, vol. 225, pp. 387-400, 2013.

[25] J. Tu and Y. You, "Random attractor of stochastic brusselator system with multiplicative noise," Discrete and Continuous Dynamical Systems - Series A, vol. 36, no. 5, pp. 2757-2779, 2016.
[26] B. Wang, "Asymptotic behavior of stochastic wave equations with critical exponents on $R_{3}$," Transactions of the American Mathematical Society, vol. 363, no. 7, pp. 3639-3663, 2011.

[27] B. Wang, "Random attractors for non-autonomous stochastic wave equations with multiplicative noise," Discrete and Continuous Dynamical Systems - Series A, vol. 34, no. 1, pp. 269-300, 2014.

[28] B. Wang, "Upper semicontinuity of random attractors for noncompact random dynamical systems," Electronic Journal of Differential Equations, vol. 2009, pp. 1-18, 2009.

[29] B. Wang, "Sufficient and necessary criteria for existence of pullback attractors for non-compact random dynamical systems," Journal of Differential Equations, vol. 253, no. 5, pp. 1544-1583, 2012.

[30] A. Goldbeter, Models for Oscillation and Excitability in Biochemical Systems, E. Segel, Ed., Mathematical Models in Molecular and Cell Biology, Cambridge University Press, Cambridge, UK, 1980. 


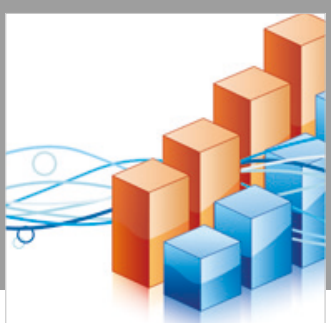

Advances in

Operations Research

\section{-n-m}
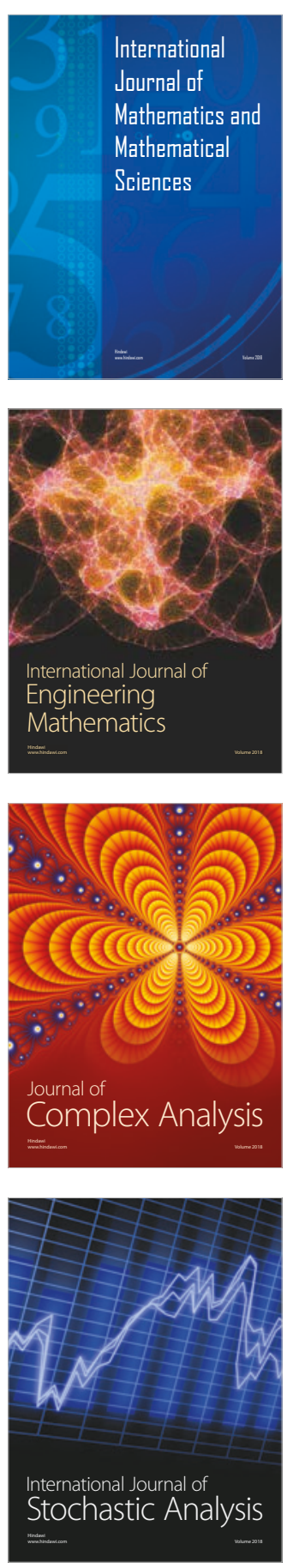
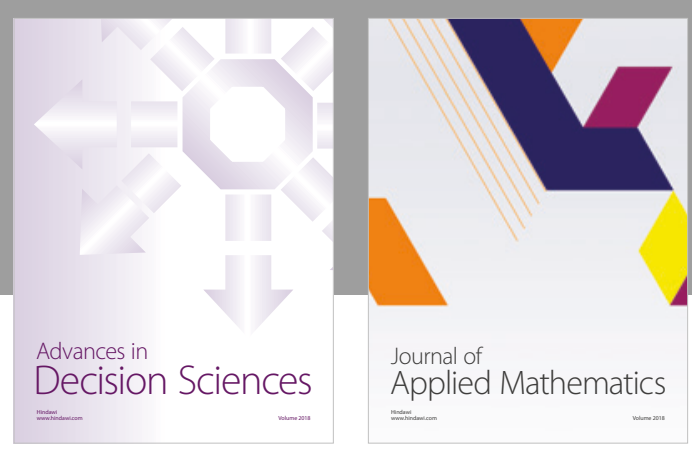

Journal of

Applied Mathematics
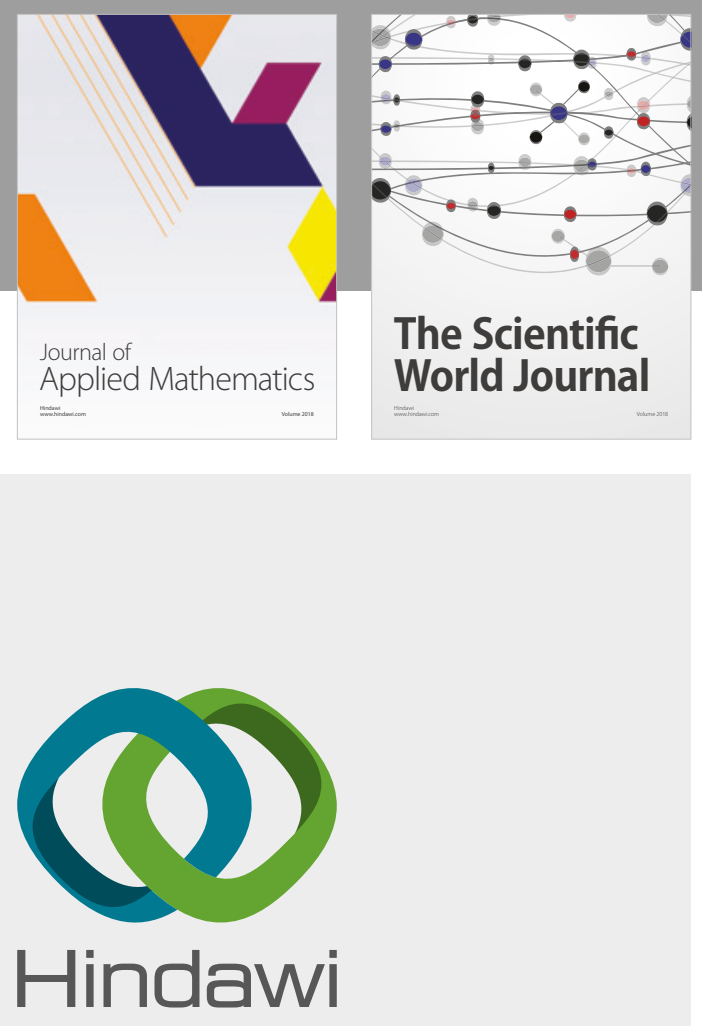

Submit your manuscripts at

www.hindawi.com

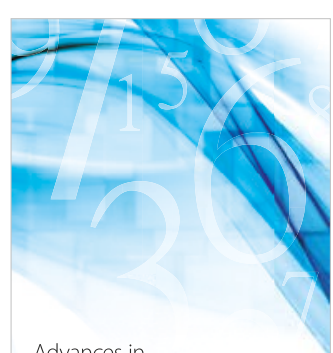

Advances in
Numerical Analysis
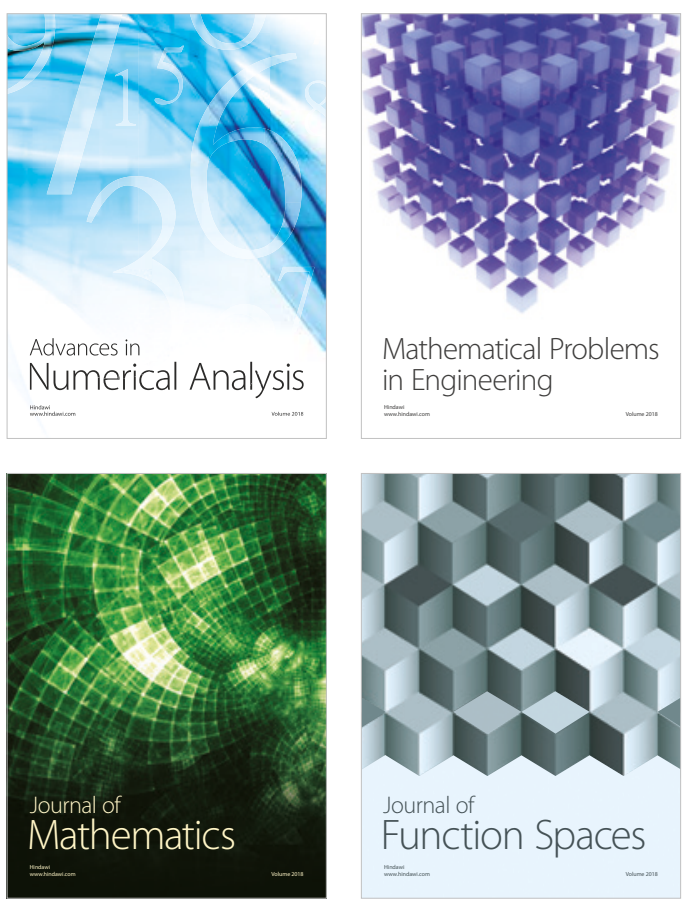

Mathematical Problems in Engineering

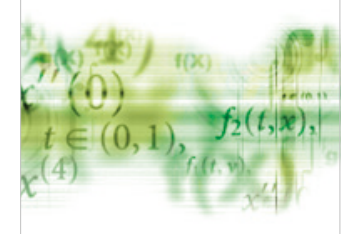

International Journal of

Differential Equations

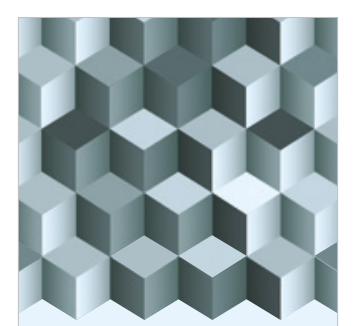

Journal of

Function Spaces
The Scientific

World Journal

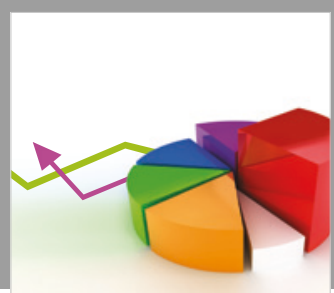

Journal of

Probability and Statistics
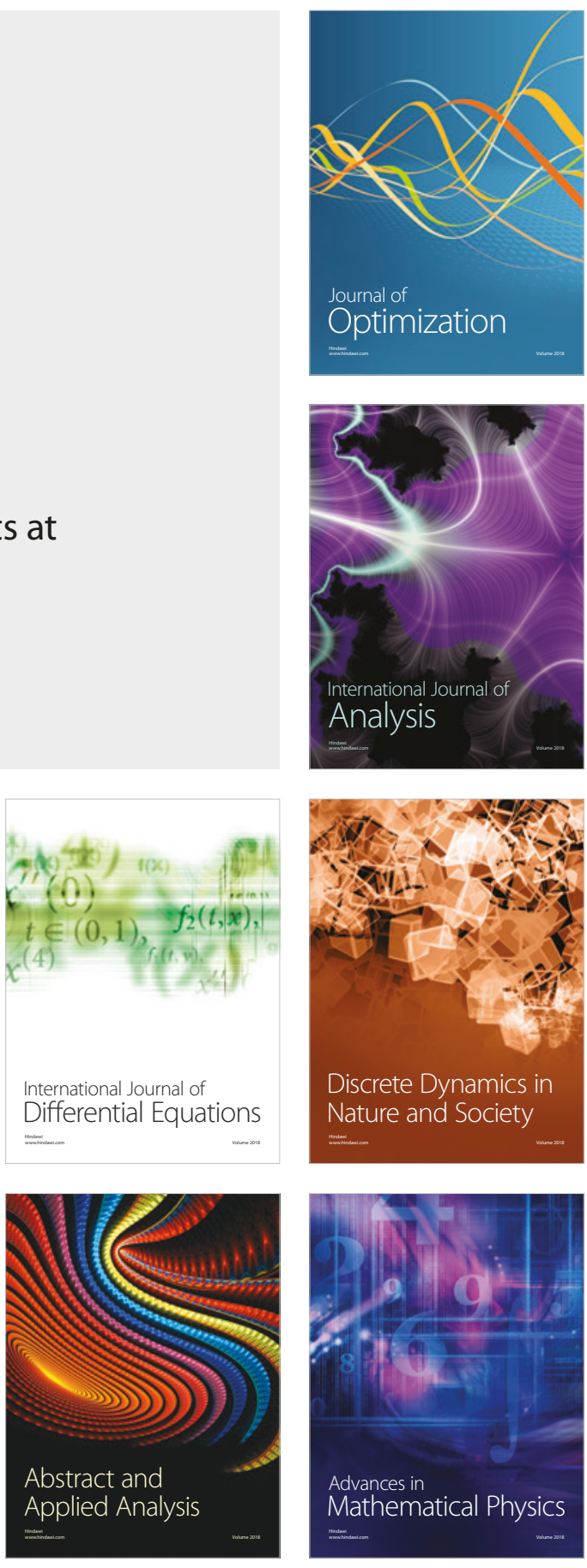